\title{
Automatically Eliminating Speculative Leaks from Cryptographic Code with Blade
}

\author{
MARCO VASSENA, CISPA Helmholtz Center for Information Security, Germany \\ CRAIG DISSELKOEN, UC San Diego, USA \\ KLAUS VON GLEISSENTHALL, Vrije Universiteit Amsterdam, Netherlands \\ SUNJAY CAULIGI, UC San Diego, USA \\ RAMI GOKKHAN KICI, UC San Diego, USA \\ RANJIT JHALA, UC San Diego, USA \\ DEAN TULLSEN, UC San Diego, USA \\ DEIAN STEFAN, UC San Diego, USA
}

We introduce BLADE, a new approach to automatically and efficiently eliminate speculative leaks from cryptographic code. BLADE is built on the insight that to stop leaks via speculative execution, it suffices to cut the dataflow from expressions that speculatively introduce secrets (sources) to those that leak them through the cache (sinks), rather than prohibit speculation altogether. We formalize this insight in a static type system that (1) types each expression as either transient, i.e., possibly containing speculative secrets or as being stable, and (2) prohibits speculative leaks by requiring that all sink expressions are stable. BLADE relies on a new abstract primitive, protect, to halt speculation at fine granularity. We formalize and implement protect using existing architectural mechanisms, and show how BLADE's type system can automatically synthesize a minimal number of protects to provably eliminate speculative leaks. We implement BLADE in the Cranelift WebAssembly compiler and evaluate our approach by repairing several verified, yet vulnerable WebAssembly implementations of cryptographic primitives. We find that BLADE can fix existing programs that leak via speculation automatically, without user intervention, and efficiently even when using fences to implement protect.

\section{CCS Concepts: • Security and privacy $\rightarrow$ Formal security models.}

Additional Key Words and Phrases: Speculative execution, Spectre, Constant-time, Type system.

\section{ACM Reference Format:}

Marco Vassena, Craig Disselkoen, Klaus von Gleissenthall, Sunjay Cauligi, Rami Gökhan Kıcı, Ranjit Jhala, Dean Tullsen, and Deian Stefan. 2021. Automatically Eliminating Speculative Leaks from Cryptographic Code with Blade. Proc. ACM Program. Lang. 5, POPL, Article 49 (January 2021), 30 pages. https://doi.org/10.1145/3434330

\section{INTRODUCTION}

Implementing secure cryptographic algorithms is hard. The code must not only be functionally correct, memory safe, and efficient, it must also avoid divulging secrets indirectly through side channels like control-flow, memory-access patterns, or execution time. Consequently, much recent

Authors' addresses: Marco Vassena, CISPA Helmholtz Center for Information Security, Germany, marco.vassena@cispa.saarland; Craig Disselkoen, UC San Diego, USA, cdisselk@cs.ucsd.edu; Klaus von Gleissenthall, Vrije Universiteit Amsterdam, Netherlands, k.freiherrvongleissenthal@vu.nl; Sunjay Cauligi, UC San Diego, USA, scauligi@eng.ucsd.edu; Rami Gökhan Kıcı, UC San Diego, USA, rkici@eng.ucsd.edu; Ranjit Jhala, UC San Diego, USA, jhala@cs.ucsd.edu; Dean Tullsen, UC San Diego, USA, tullsen@cs.ucsd.edu; Deian Stefan, UC San Diego, USA, deian@cs.ucsd.edu.

This work is licensed under a Creative Commons Attribution-ShareAlike 4.0 International License.

(C) 2021 Copyright held by the owner/author(s).

2475-1421/2021/1-ART49

https://doi.org/10.1145/3434330

Proc. ACM Program. Lang., Vol. 5, No. POPL, Article 49. Publication date: January 2021. 
work focuses on how to ensure implementations do not leak secrets e.g., via type systems [Cauligi et al. 2019; Watt et al. 2019], verification [Almeida et al. 2016; Protzenko et al. 2019], and program transformations [Barthe et al. 2019].

Unfortunately, these efforts can be foiled by speculative execution. Even if secrets are closely controlled via guards and access checks, the processor can simply ignore these checks when executing speculatively. This, in turn, can be exploited by an attacker to leak protected secrets.

In principle, memory fences block speculation, and hence, offer a way to recover the original security guarantees. In practice, however, fences pose a dilemma. Programmers can restore security by conservatively inserting fences after every load (e.g., using Microsoft's Visual Studio compiler pass [Donenfeld 2020]), but at huge performance costs. Alternatively, they can rely on heuristic approaches for inserting fences [Wang et al. 2018], but forgo guarantees about the absence of side-channels. Since missing even one fence can allow an attacker to leak any secret from the address space, secure runtime systems-in particular, browsers like Chrome and Firefox-take yet another approach and isolate secrets from untrusted code in different processes to avoid the risk altogether [Blog 2010; Mozilla Wiki 2018]. Unfortunately, the engineering effort of such a multi-process redesign is huge-e.g., Chrome's redesign took five years and roughly $450 \mathrm{~K}$ lines of code changes [Reis et al. 2019].

In this paper, we introduce BLADE, a new, fully automatic approach to provably and efficiently eliminate speculation-based leakage from constant-time cryptographic code. BLADE is based on the key insight that to prevent leaking data via speculative execution, it is not necessary to stop all speculation. Instead, it suffices to cut the data flow from expressions (sources) that could speculatively introduce secrets to those that leak them through the cache (sinks). We develop this insight into an automatic enforcement algorithm via four contributions.

A JIT-Step Semantics for Speculation. A key aim of BLADE is to enable source-level reasoning about the absence of speculation-based information leaks. This is crucial to let the source-level type system use control- and data-flow information to optimally prevent leaks. High-level reasoning requires a source-level semantic model of speculation which has, so far, proven to be challenging as the effects of speculation manifest at the very low machine-level: e.g., as branch-prediction affects the streams of low-level instructions that are fetched and (speculatively) executed. We address this challenge with our first contribution: a JIT-step semantics for a high-level WHILE language that lets us reconcile the tension between high-level reasoning and low-level speculation. These semantics translate high-level commands to low-level machine instructions in a just-in-time (JIT) fashion, whilst tracking control-flow predictions at branch points and modeling the speculative execution path of the program as well as the "paths not taken" as stacks of high-level commands.

Our low-level instructions are inspired by a previous formal model of a speculative and out-oforder processor [Cauligi et al. 2020], and let us model the essence of speculation-based attacks-in particular Spectre-PHT [Canella et al. 2019; Kiriansky and Waldspurger 2018; Kocher et al. 2019]-by modeling precisely how speculation can occur and what an attacker can observe via speculation (§ 3). To prevent leakage, we propose and formalize the semantics of an abstract primitive called protect that embodies several hardware mechanisms proposed in recent work [Taram et al. 2019; $\mathrm{Yu}$ et al. 2019]. Crucially, and in contrast to a regular fence which stops all speculation, protect only stops speculation for a given variable. For example $x:=\operatorname{protect}(e)$ ensures that the value of $e$ is assigned to $x$ only after $e$ has been assigned its stable, non-speculative value. Though we encourage hardware vendors to implement protect in future processors, for backwards compatibility, we implement and evaluate two versions of protect on existing hardware-one using fences, another using speculative load hardening (SLH) [Carruth 2019]. 
A Type System for Speculation. Our second contribution is an approach to conservatively approximating the dynamic semantics of speculation via a static type sytem that types each expression as either transient (T), i.e., expressions that may contain speculative secrets, or stable (S), i.e., those that cannot (§ 4.1). Our system prohibits speculative leaks by requiring that all sink expressions that can influence intrinsic attacker visible behavior (e.g., cache addresses) are typed as stable The type system does not rely on user-provided security annotations to identify sensitive sources and public sinks. Instead, we conservatively reject programs that exhibit any flow of information from transient sources to stable sinks. This, in turn, allows us to automatically identify speculative vulnerabilities in existing cryptographic code (where secrets are not explicitly annotated). We connect the static and dynamic semantics by proving that well-typed constant-time programs are secure, i.e., they are also speculative constant-time [Cauligi et al. 2020] (§5). This result extends the pre-existing guarantees about sequential constant-time execution of verified cryptographic code to our speculative execution model.

Automatic Protection. Existing programs that are free of protect statements are likely insecure under speculation (see Section 7 and [Cauligi et al. 2020]) and will be rejected by our type system. Thus, our third contribution is an algorithm that finds potential speculative leaks and automatically synthesizes a minimal number of protect statements to ensure that the program is speculatively constant-time ( $(4.2)$. To this end, we extend the type checker to construct a def-use graph that captures the data-flow between program expressions. The presence of a path from transient sources to stable sinks in the graph indicates a potential speculative leak in the program. To repair the program, we only need to identify a cut-set, a set of variables whose removal eliminates all the leaky paths in the graph. We show that inserting a protect statement for each variable in a cut-set suffices to yield a program that is well-typed, and hence, secure with respect to speculation. Finding such cuts is an instance of the classic Max-Flow/Min-Cut problem, so existing polynomial time algorithms let us efficiently synthesize protect statements that resolve the dilemma of enforcing security with minimal number of protections.

BLADE Tool. Our final contribution is an automatic push-button tool, BLADE, which eliminates potential speculative leaks using this min-cut algorithm. BLADE extends the Cranelift compiler [Bytecode Alliance 2020], which compiles WebAssembly (Wasm) to x86 machine code; thus, BLADE operates on programs expressed in Wasm. However, operating on Wasm is not fundamental to our approach-we believe that BLADE's techniques can be applied to other programming languages and bytecodes.

We evaluate BLADE by repairing verified yet vulnerable (to transient execution attacks) constanttime cryptographic primitives from Constant-time Wasm (CT-Wasm) [Watt et al. 2019] and $\mathrm{HACL}^{*}$ [Zinzindohoué et al. 2017] (§ 7). Compared to existing fully automatic speculative mitigation approaches (as notably implemented in Clang), BLADE inserts an order of magnitude fewer protections (fences or SLH masks). BLADE's fence-based implementation imposes modest performance overheads: (geometric mean) 5.0\% performance overhead on our benchmarks to defend from Spectre v1, or 15.3\% overhead to also defend from Spectre v1.1. Both results are significant gains over current solutions. Our fence-free implementation, which automatically inserts SLH masks, is faster in the Spectre v1 case-geometric mean $1.7 \%$ overhead-but slower when including Spectre v1.1 protections, imposing $26.6 \%$ overhead.

\section{OVERVIEW}

This section gives a brief overview of the kinds of speculative leaks that BLADE identifies and how it repairs such programs by careful placement of protect statements. We then describe how BLADE 


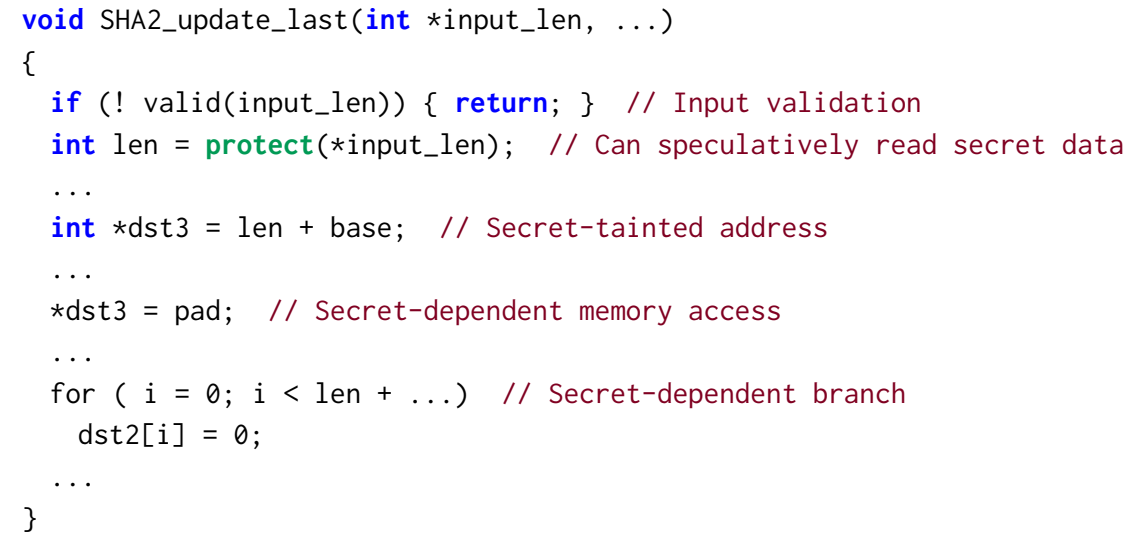

Fig. 1. Code fragment adapted from the $\mathrm{HACL}^{*} \mathrm{SHA} 2$ implementation, containing two potential speculative execution vulnerabilities: one through the data cache by writing memory at a secret-tainted address, and one through the instruction cache via a secret-tainted control-flow dependency. The patch computed by BLADE is shown in green.

(1) automatically repairs existing programs using our minimal protect inference algorithm and (2) proves that the repairs are correct using our transient-flow type system.

\subsection{Two Kinds of Speculative Leaks}

Figure 1 shows a code fragment of the SHA2_update_last function, a core piece of the SHA2 cryptographic hash function implementation, adapted (to simplify exposition) from the HACL* library. This function takes as input a pointer input_len, validates the input (line 3), loads from memory the public length of the hash (line 4, ignore protect for now), calculates a target address dst3 (line 6), and pads the buffer pointed to by dst3 (line 8). Later, it uses len to determine the number of initialization rounds in the condition of the for-loop on line 10.

Leaking Through a Memory Write. During normal, sequential execution this code is not a problem: the function validates the input to prevent classic buffer-overflow vulnerabilities. However, during speculation, an attacker can exploit this function to leak sensitive data. To do this, the attacker first has to mistrain the branch predictor to predict the next input to be valid. Since input_len is a function parameter, the attacker can do this by, e.g., calling the function repeatedly with legitimate addresses. After mistraining the branch predictor this way, the attacker manipulates input_len to point to an address containing secret data and calls the function again, this time with an invalid pointer. As a result of the mistraining, the branch predictor causes the processor to skip validation and load the secret into len, which in turn is used to calculate pointer dst3. The location pointed to by dst 3 is then written on line 8 , leaking the secret data. Even though pointer dst 3 is invalid and the subsequent write will not be visible at the program level (the processor disregards it), the side-effects of the memory access persist in the cache and therefore become visible to the attacker. In particular, the attacker can extract the target address-and thereby the secret-using cache timing measurements [Ge et al. 2018].

Leaking Through a Control-Flow Dependency. The code in Figure 1 contains a second potential speculative vulnerability, which leaks secrets through a control-flow dependency instead of a memory access. To exploit this vulnerability, the attacker can similarly manipulate the pointer input_len to point to a secret after mistraining the branch predictor to skip validation. But instead of leaking 
the secret directly through the data cache, the attacker can leak the value indirectly through a control-flow dependency: in this case, the secret determines how often the initialization loop (line 10) is executed during speculation. The attacker can then infer the value of the secret from a timing attack on the instruction cache or (much more easily) on iteration-dependent lines of the data cache.

\subsection{Eliminating Speculative Leaks}

Preventing the Leak using Memory Fences. Since these leaks exploit the fact that input validation is speculatively skipped, we can prevent them by making sure that dangerous operations such as the write on line 8 or the loop condition check on line 10 are not executed until the input has been validated. Intel [2018a], AMD [2018], and others [Donenfeld 2020; Pardoe 2018] recommend doing this by inserting a speculation barrier after critical validation check-points. This would place a memory fence after line 3, but anywhere between lines 3 and 8 would work. This fence would stop speculation over the fence: statements after the fence will not be executed until all statements up to the fence (including input validation) executed. While fences can prevent leaks, using fences as such is more restrictive than necessary-they stop speculative execution of all following instructions, not only of the instructions that leak-and thus incur a high performance cost [Taram et al. 2019; Tkachenko 2018].

Preventing the Leak Efficiently. We do not need to stop all speculation to prevent leaks. Instead, we only need to ensure that potentially secret data, when read speculatively, cannot be leaked. To this end, we propose an alternative way to stop speculation from reaching the operations on line 8 and line 10, through a new primitive called protect. Rather than eliminate all speculation, protect only stops speculation along a particular data-path. We use protect to patch the program on line 4. Instead of assigning the value len directly from the result of the load, the memory load is guarded by a protect statement. This ensures that the value assigned to len is always guaranteed to use the input_len pointer's final, nonspeculative value. This single protect statement on line 4 is sufficient to fix both of the speculative leaks described in Section 2.1-it prevents any speculative, secret data from reaching lines 8 or 10 where it could be leaked to the attacker.

Implementation of protect. Our protect primitive provides an abstract interface for fine grained control of speculation. This allows us to eliminate speculation-based leaks precisely and only when needed. However, whether protect can eliminate leaks with tolerable runtime overhead depends on its concrete implementation. We consider and formalize two implementations: an ideal implementation and one we can implement on today's hardware.

To have fine grain control of speculation, protect must be implemented in hardware and exposed as part of the ISA. Though existing processors provide only coarse grained control over speculation through memory fence instructions, this might change in the future. For example, recently proposed microprocessor designs [Taram et al. 2019; Yu et al. 2019] provide new hardware mechanisms to control speculation, in particular to restrict targeted types of speculation while allowing other speculation to proceed: this suggests that protect could be implemented efficiently in hardware in the future.

Even if future processors implement protect, we still need to address Spectre attacks on existing hardware. Hence, we formalize and implement protect in software, building on recent Spectre attack mitigations [Schwarz et al. 2020]. Specifically, we propose a self-contained approach inspired by Clang's Speculative Load Hardening (SLH) [Carruth 2019]. At a high level, Clang's SLH stalls speculative load instructions in a conditional block by inserting artificial data-dependencies between loaded addresses and the value of the condition. This ensures that the load is not executed before the branch condition is resolved. Unfortunately, this approach unnecessarily stalls all non-constant conditional load instructions, regardless of whether they can influence a subsequent load and thus can actually cause speculative data leaks. Furthermore, this approach is unsound-it can also miss some speculative leaks, e.g., if a load instruction is not in the same conditional block that validates its 


$$
\begin{aligned}
& \text { EXAMPLE } \\
& \hline x:=a\left[i_{1}\right] \\
& y:=a\left[i_{2}\right] \\
& z:=x+y \\
& w:=b[z]
\end{aligned}
$$

$$
\begin{aligned}
& \text { EXAMPLE PATCHED } \\
& \hline x:=\operatorname{protect}\left(a\left[i_{1}\right]\right) \\
& y:=\operatorname{protect}\left(a\left[i_{2}\right]\right) \\
& z:=\operatorname{protect}(x+y) \\
& w:=b[z]
\end{aligned}
$$

Fig. 2. Running example. Program EXAMPLE is shown on the left and the patched program is shown on the right. The orange patch is sub-optimal because it requires more protect statements than the optimal green patch.

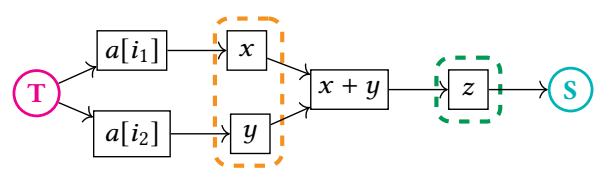

Fig. 3. Subset of the def-use graph of ExAMPLE. The dashed lines identify two valid choices of cut-sets. The left cut requires removing two nodes and thus inserting two protect statements. The right cut shows a minimal solution, which only requires removing a single node.

address (like the code in Figure 1). In contrast to Clang, our approach applies SLH selectively, only to individual load instructions whose result flows to an instruction which might leak, and precisely, by using accurate bounds-check conditions to ensure that only data from valid addresses can be loaded.

\subsection{Automatically Repairing Speculative Leaks via protect-Inference}

BLADE automatically finds potential speculative leaks and synthesizes a minimal number of protect statements to eliminate the leaks. We illustrate this process using the simple program ExAmPLE in Figure 2 as a running example. The program reads two values from an array $\left(x:=a\left[i_{1}\right]\right.$ and $\left.y:=a\left[i_{2}\right]\right)$, adds them $(z:=x+y)$, and indexes another array with the result $(w:=b[z])$. This program is written using our formal calculus in which all array operations are implicitly bounds-checked and thus no explicit validation code is needed.

Like the SHA2 example from Figure 1, ExAMPLE contains a speculative execution vulnerability: the speculative array reads could bypass their bounds checks and so $x$ and $y$ can contain transient secrets (i.e., secrets introduced by misprediction). This secret data then flows to $z$, and finally leaks through the data cache when reading $b[z]$.

Def-Use Graph. To secure the program, we need to cut the dataflow between the array reads which could introduce transient secret values into the program, and the index in the array read where they are leaked through the cache. For this, we first build a def-use graph whose nodes and directed edges capture the data dependencies between the expressions and variables of a program. For example, consider (a subset of) the def-use graph of program ExAMPLE in Figure 3. In the graph, the edge $x \rightarrow x+y$ indicates that $x$ is used to compute $x+y$. To track how transient values propagate in the def-use graph, we extend the graph with the special node T, which represents the source of transient values of the program. Since reading memory creates transient values, we connect the $\mathrm{T}$ node to all nodes containing expressions that explicitly read memory, e.g., $\mathbf{T} \rightarrow a\left[i_{1}\right]$. Following the data dependencies along the edges of the def-use graph, we can see that node $T$ is transitively connected to node $z$, which indicates that $z$ can contain transient data at runtime. To detect insecure uses of transient values, we then extend the graph with the special node $\mathrm{S}$, which represents the sink of stable (i.e., non-transient) values of a program. Intuitively, this node draws all the values of a program that must be stable to avoid transient execution attacks. Therefore, we 
connect all expression used as array indices in the program to the S node, e.g., $z \rightarrow \mathrm{S}$. The fact that the graph in Figure 3 contains a path from $T$ to $S$ indicates that transient data flows through data dependencies into (what should be) a stable index expression and thus the program may be leaky.

Cutting the Dataflow. In order to make the program safe, we need to cut the data-flow between $\mathrm{T}$ and $\mathrm{S}$ by introducing protect statements. This problem can be equivalently restated as follows: find a cut-set, i.e., a set of variables, such that removing the variables from the graph eliminates all paths from $\mathrm{T}$ to $\mathrm{S}$. Each choice of cut-set defines a way to repair the program: simply add a protect statement for each variable in the set. Figure 3 contains two choices of cut-sets, shown as dashed lines. The cut-set on the left requires two protect statements, for variables $x$ and $y$ respectively, corresponding to the orange patch in Figure 2. The cut-set on the right is minimal, it requires only a single protect, for variable $z$, and corresponds to the green patch in Figure 2. Intuitively, minimal cut-sets result in patches that introduce as few protects as needed and therefore allow more speculation. Luckily, the problem of finding a minimal cut-set is an instance of the classic Min-Cut/Max-Flow problem, which can be solved using efficient, polynomial-time algorithms [Ford and Fulkerson 2010]. For simplicity, BLADE adopts a uniform cost model and therefore synthesizes patches that contain a minimal number of protect statements, regardless of their position in the code and how many times they can be executed. Though our evaluation shows that even this simple model imposes modest overheads ( $\$ 7)$, our implementation can be easily optimized by considering additional criteria when searching for a minimal cut set, with further performance gain likely. For example, we could assign weights proportional to execution frequency, or introduce penalties for placing protect inside loops.

\subsection{Proving Correctness via Transient-Flow Types}

To ensure that we add protect statements in all the right places (without formally verifying our repair algorithm), we use a type system to prove that patched programs are secure, i.e., they satisfy a semantic security condition. The type system simplifies the security analysis-we can reason about program execution directly rather than through generic flows of information in the def-use graph. Moreover, restricting the security analysis to the type system makes the security proofs independent of the specific algorithm used to compute the program repairs (e.g., the Max-Flow/MinCut algorithm). As long as the repaired program type checks, BlADE's formal guarantees hold. To show that the patches obtained from cutting the def-use graph of a given program are correct (i.e., they make the program well-typed), our transient-flow type system constructs its def-use graph from the type-constraints generated during type inference.

Typing Judgement. Our type system statically assigns a transient-flow type to each variable: a variable is typed as transient (written as T), if it can contain transient data (i.e., potential secrets) at runtime, and as stable (written as S), otherwise. For instance, in program ExAmpLE (Fig. 3) variables $x$ and $y$ (and hence $z$ ) are typed as transient because they may temporarily contain secret data originated from speculatively reading the array out of bounds. Given a typing environment $\Gamma$ which assigns a transient-flow type to each variable, and a command $c$, the type system defines a judgement $\Gamma \vdash c$ saying that $c$ is free of speculative execution bugs ( $(4.1)$. The type system ensures that transient expressions are not used in positions that may leak their value by affecting memory reads and writes, e.g., they may not be used as array indices and in loop conditions. Additionally, it ensures that transient expressions are not written to stable variables, except via protect. For example, our type system rejects program EXAMPLE because it uses transient variable $z$ as an array index, but it accepts program ExAmple PATched in which $z$ can be typed stable thanks to the protect statements. We say that variables whose assignment is guarded by a protect statement (like $z$ in ExAmple PATched) are "protected variables". 
To study the security guarantees of our type system, we define a JIT-step semantics for speculative execution of a high-level WHILE language (§ 3), which resolves the tension between source-level reasoning and machine-level speculation. We then show that our type system indeed prevents speculative execution attacks, i.e., we prove that well-typed programs remain constant-time under the speculative semantics (§5).

Type Inference. Given an input program, we construct the corresponding def-use graph by collecting the type constraints generated during type inference. Type inference is formalized by a typing-inference judgment $\Gamma$, Prot $\vdash c \Rightarrow k(\S 4.2)$, which extends the typing judgment from above with (1) a set of implicitly protected variables Prot (the cut-set), and (2) a set of type-constraints $k$ (the def-use graph). Intuitively, the set Prot identifies the variables of program $c$ that contain transient data and that must be protected (i.e., they must be made stable using protect) to avoid leaks. At a high level, type inference has 3 steps: (i) generate a set of constraints under an initial typing environment and protected set that allow any program to type-check, (ii) construct the def-use graph from the constraints and find a cut-set (the final protected set), and (iii) compute the final typing environment which types the variables in the cut-set as stable. To characterize the security of a still unrepaired program after type inference, we define a typing judgment $\Gamma$, Prot $\vdash c$, where unprotected variables are explicitly accounted for in the Prot set. ${ }^{1}$ Intuitively, the program is secure if we promise to insert a protect statement for each variable in Prot. To repair programs, we simply honor this promise and insert a protect statement for each variable in the protected set of the typing judgment obtained above. Once repaired, the program type checks under an empty protected set.

\subsection{Attacker Model}

We delineate the extents of the security guarantees of our type system and repair algorithm by discussing the attacker model considered in this work. We assume an attacker model where the attacker runs cryptographic code, written in Wasm, on a speculative out-of-order processor; the attacker can influence how programs are speculatively executed using the branch predictor and choose the instruction execution order in the processor pipeline. The attacker can make predictions based on control-flow history and memory-access patterns similar to real, adaptive predictors. Though these predictions can depend on secret information in general, we assume that they are secret-independent for the constant-time programs repaired by BLADE.

We assume that the attacker can observe the effects of their actions on the cache, even if these effects are otherwise invisible at the ISA level. In particular, while programs run, the attacker can take precise timing measurements of the data- and instruction-cache with a cache-line granularity, and thus infer the value of secret data. These features allow the attacker to mount Spectre-PHT attacks [Kiriansky and Waldspurger 2018; Kocher et al. 2019] and exfiltrate data through FLUSH+RELOAD [Yarom and Falkner 2014] and PRIME+PROBE [Tromer et al. 2010] cache side-channel attacks. We do not consider speculative attacks that rely on the Return Stack Buffer (e.g., Spectre-RSB [Koruyeh et al. 2018; Maisuradze and Rossow 2018]), Branch Target Buffer (Spectre-BTB [Kocher et al. 2019]), or Store-to-Load forwarding misprediction (Spectre-STL [Horn 2018], recently reclassified as a Meltdown attack [Moghimi et al. 2020]). We similarly do not consider Meltdown attacks [Lipp et al. 2018] or attacks that do not use the cache to exfiltrate data, e.g., port contention (SMoTherSpectre [Bhattacharyya et al. 2019]).

\section{A JIT-STEP SEMANTICS FOR SPECULATION}

We now formalize the concepts discussed in the overview, presenting a semantics in this section and a type system in Section 4. Our semantics are explicitly conservative and abstract, i.e., they only

\footnotetext{
${ }^{1}$ The judgment $\Gamma \vdash c$ is just a short-hand for $\Gamma, \varnothing \vdash c$.
} 
Values $v::=n|b| a$

Expr. $e:=v|x| e+e \mid e<e$

$|e \otimes e| e ? e: e$

| length $(e) \mid$ base $(e)$

Rhs. $r:=e|* e| a[e]$

Cmd. $c:=$ skip $|x:=r| * e=e$

$|a[e]:=e|$ fail $\mid c ; c$

$\mid$ if $e$ then $c$ else $c$

| while $e$ do $c$

$\mid x:=\operatorname{protect}(r)$

(a) Source syntax.
Instructions $i::=\operatorname{nop}|x:=e| x:=\operatorname{load}(e)$

$|\operatorname{store}(e, e)| x:=\operatorname{protect}(e)$

$\left|\operatorname{guard}\left(e^{b}, c s, p\right)\right| \operatorname{fail}(p)$

Directives $d::=$ fetch $\mid$ fetch $b \mid$ exec $n \mid$ retire

Observations $o::=\epsilon|\operatorname{read}(n, p s)| \operatorname{write}(n, p s)$

$|\operatorname{fail}(p)| \operatorname{rollback}(p)$

Predictions $b \in\{$ true, false $\}$

Guard Fail Ids. $p \in \mathbb{N}$

Instr. Buffers is ::= $i:$ is | []

Cmd Stacks $c s::=c: c s \mid[]$

Stores $\mu \in \mathbb{N} \rightarrow$ Value

Var. Maps $\rho \in$ Var $\rightarrow$ Value

Config. $C::=\langle i s, c s, \mu, \rho\rangle$

(b) Processor syntax.

Fig. 4. Syntax of the calculus.

model the essential features required to capture Spectre-PHT attacks on modern microarchitecturesnamely, speculative and out-of-order execution. We do not model microarchitectural features exploited by other attacks (e.g., Spectre-BTB and Spectre-RSB), which require a more complex semantics model [Cauligi et al. 2020; Guanciale et al. 2020].

Language. We start by giving a formal just-in-time step semantics for a WHILE language with speculative execution. We present the language's source syntax in Figure 4a. Its values consist of Booleans $b$, pointers $n$ represented as natural numbers, and arrays $a$. The calculus has standard expression constructs: values $v$, variables $x$, addition $e+e$, and a comparison operator $e<e$. To formalize the semantics of the software (SLH) implementation of protect $(\cdot)$ we rely on several additional constructs: the bitwise AND operator $e \otimes e$, the non-speculative conditional ternary operator $e$ ? $e: e$, and primitives for getting the length (length(.)) and base address $($ base $(\cdot))$ of an array. We do not specify the size and bit-representation of values and, instead, they satisfy standard properties. In particular, we assume that $n \otimes \mathbf{0}=\mathbf{0}=\mathbf{0} \otimes n$, i.e., the bitmask $\mathbf{0}$ consisting of all $0 \mathrm{~s}$ is the zero element for $\otimes$ and corresponds to number 0 , and $n \otimes 1=n=1 \otimes n$, i.e., the bitmask 1 consisting of all $1 \mathrm{~s}$ is the unit element for $\otimes$ and corresponds to some number $n^{\prime} \neq 0$. Commands include variable assignments, pointer dereferences, array stores, ${ }^{2}$ conditionals, and loops. ${ }^{3}$ Beyond these standard constructs, we expose a special command that is used to prevent transient execution attacks: the command $x:=\operatorname{protect}(r)$ evaluates $r$ and assigns its value to $x$, but only after the value

\footnotetext{
${ }^{2}$ The syntax for reading and writing arrays requires the array to be a constant value (i.e., $a[e]$ ) to simplify our security analysis. This simplification does not restrict the power of the attacker, who can still access memory at arbitrary addresses speculatively through the index expression $e$.

${ }^{3}$ The branch constructs of our source language can be used to directly model Wasm's branch constructs (e.g., conditionals and while loops can map to Wasm's br_if and br) or could be easily extended (e.g., we can add switch statements to support Wasm's branch table br_table instruction) [Haas et al. 2017]. To keep our calculus small, we do not replicate Wasm's (less traditional) low-level branch constructs which rely on block labels (e.g., br label). Since Wasm programs are statically typed, these branch instructions switch execution to statically known, well-specified program points marked with labels. Our calculus simply avoids modeling labels explicitly and replaces them with the code itself.
} 
is stable (i.e., non-transient). Lastly, fail triggers a memory violation error (caused by trying to read or write an array out-of-bounds) and aborts the program.

JIT-Step Semantics. Our operational semantics formalizes the execution of source programs on a pipelined processor and thus enables source-level reasoning about speculation-based information leaks. In contrast to previous semantics for speculative execution [Cauligi et al. 2020; Cheang et al. 2019; Guarnieri et al. 2020; McIlroy et al. 2019], our processor abstract machine does not operate directly on fully compiled assembly programs. Instead, our processor translates highlevel commands into low-level instructions just in time, by converting individual commands into corresponding instructions in the first stage of the processor pipeline. To support this execution model, the processor progressively flattens structured commands (e.g., if-statements and while loops) into predicted straight-line code and maintains a stack of (partially flattened) commands to keep track of the program execution path. In this model, when the processor detects a misspeculation, it only needs to replace the command stack with the sequence of commands that should have been executed instead to restart the execution on the correct path.

Processor Instructions. Our semantics translates source commands into an abstract set of processor instructions shown in Figure 4b. Most of the processor instructions correspond directly to the basic source commands. Notably, the processor instructions do not include an explicit jump instruction for branching. Instead, a sequence of guard instructions represents a series of pending branch points along a single predicted path. Guard instructions have the form guard $\left(e^{b}, c s, p\right)$, which records the branch condition $e$, its predicted truth value $b$, and a unique guard identifier $p$, used in our security analysis (Section 5). Each guard attests to the fact that the current execution is valid only if the branch condition gets resolved as predicted. In order to enable a roll-back in case of a missprediction, guards additionally record the sequence of commands cs along the alternative branch.

Directives and Observations. Instructions do not have to be executed in sequence: they can be executed in any order, enabling out-of-order execution. We use a simple three stage processor pipeline: the execution of each instruction is split into fetch, exec, and retire. We do not fix the order in which instructions and their individual stages are executed, nor do we supply a model of the branch predictor to decide which control flow path to follow. Instead, we let the attacker supply those decisions through a set of directives [Cauligi et al. 2020] shown in Fig. 4b. For example, directive fetch true fetches the true branch of a conditional and exec $n$ executes the $n$-th instruction in the reorder buffer. Executing an instruction generates an observation (Fig. 4b) which records attacker observable behavior. Observations include speculative memory reads and writes (i.e., $\operatorname{read}(n, p s)$ and write $(n, p s)$ issued while the guard and fail instructions identified by $p s$ are pending), rollbacks (i.e., $\operatorname{rollback}(p)$ due to misspeculation of guard $p$ ), and memory violations (i.e., fail $(p)$ due to instruction fail $(p))$. Most instructions generate the silent observation $\epsilon$. Like [Cauligi et al. 2020], we do not include observations about branch directions. Doing so would not increase the power of the attacker: a constant-time program that leaks through the branch direction would also leak through the presence or absence of rollback events, i.e., different predictions produce different rollback events, capturing leaks due to branch direction.

Configurations and Reduction Relation. We formally specify our semantics as a reduction relation between processor configurations. A configuration $\langle i s, c s, \mu, \rho\rangle$ consists of a queue of inflight instructions is called the reorder buffer, a stack of commands cs representing the current execution path, a memory $\mu$, and a map $\rho$ from variables to values. A reduction step $C \stackrel{d}{\rightarrow}{ }_{o} C^{\prime}$ denotes that, under directive $d$, configuration $C$ is transformed into $C^{\prime}$ and generates observation $o$. To execute a program $c$ with initial memory $\mu$ and variable map $\rho$, the processor initializes the configuration with an empty reorder buffer and inserts the program into the command stack, 


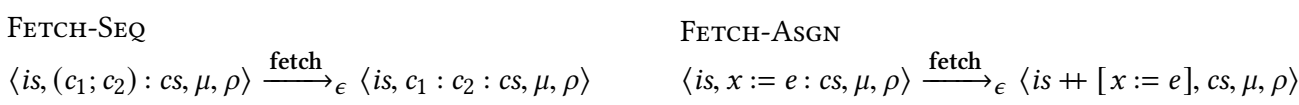

FETCH-PTR-LOAD

$\langle i s, x:=* e: c s, \mu, \rho\rangle \stackrel{\text { fetch }}{\longrightarrow}_{\epsilon}\langle i s+[x:=\operatorname{load}(e)], c s, \mu, \rho\rangle$

FETCH-ARRAY-LOAD

$\frac{c=x:=a[e] \quad e_{1}=e<\operatorname{length}(a) \quad e_{2}=\operatorname{base}(a)+e \quad c^{\prime}=\text { if } e_{1} \text { then } x:=* e_{2} \text { else fail }}{\langle i s, c: c s, \mu, \rho\rangle \stackrel{\text { fetch }}{\longrightarrow}\left\langle i s, c^{\prime}: c s, \mu, \rho\right\rangle}$

FETCH-IF-True

$\frac{c=\text { if } e \text { then } c_{1} \text { else } c_{2} \quad \operatorname{fresh}(p) \quad i=\operatorname{guard}\left(e^{\text {true }}, c_{2}: c s, p\right)}{\langle i s, c: c s, \mu, \rho\rangle \stackrel{\text { fetch true }}{\longrightarrow}\left\langle i s+[i], c_{1}: c s, \mu, \rho\right\rangle}$

Fig. 5. Fetch stage (selected rules).

i.e., $\langle[],[c], \mu, \rho\rangle$. Then, the execution proceeds until both the reorder buffer and the stack in the configuration are empty, i.e., we reach a configuration of the form $\left\langle[],[], \mu^{\prime}, \rho^{\prime}\right\rangle$, for some final memory store $\mu^{\prime}$ and variable map $\rho^{\prime}$.

We now discuss the semantics rules of each execution stage and then those for our security primitive.

\subsection{Fetch Stage}

The fetch stage flattens the input commands into a sequence of instructions which it stores in the reorder buffer. Figure 5 presents selected rules; the remaining rules are in the extended version of this paper [Vassena et al. 2020]. Rule [Fetch-SEQ] pops command $c_{1} ; c_{2}$ from the commands stack and pushes the two sub-commands for further processing. [Fetch-AsGN] pops an assignment from the commands stack and appends the corresponding processor instruction $(x:=e)$ at the end of the reorder buffer. ${ }^{4}$ Rule [FETCH-PTR-LOAD] is similar and simply translates pointer dereferences to the corresponding load instruction. Arrays provide a memory-safe interface to read and write memory: the processor injects bounds-checks when fetching commands that read and write arrays. For example, rule [FETCH-ARRAY-LOAD] expands command $x:=a[e]$ into the corresponding pointer dereference, but guards the command with a bounds-check condition. First, the rule generates the condition $e_{1}=e<\operatorname{length}(a)$ and calculates the address of the indexed element $e_{1}=$ base $(a)+e$. Then, it replaces the array read on the stack with command if $e_{1}$ then $x:=* e_{2}$ else fail to abort the program and prevent the buffer overrun if the bounds check fails. Later, we show that speculative outof-order execution can simply ignore the bounds check guard and cause the processor to transiently read memory at an invalid address. Rule [FетсH-IF-True] fetches a conditional branch from the stack and, following the prediction provided in directive fetch true, speculates that the condition $e$ will evaluate to true. Thus, the processor inserts the corresponding instruction guard $\left(e^{\text {true }}, c_{2}: c s, p\right)$ with a fresh guard identifier $p$ in the reorder buffer and pushes the then-branch $c_{1}$ onto the stack cs. Importantly, the guard instruction stores the else-branch together with a copy of the current commands stack (i.e., $c_{2}: c s$ ) as a rollback stack to restart the execution in case of misprediction.

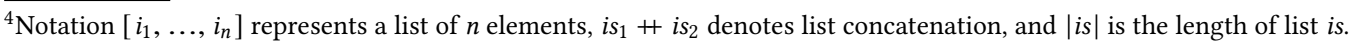




$$
\begin{aligned}
& \phi(\rho,[])=\rho \\
& \phi(\rho,(x:=v): i s)=\phi(\rho[x \mapsto v], i s) \\
& \phi(\rho,(x:=e): i s)=\phi(\rho[x \mapsto \perp], i s) \\
& \phi(\rho,(x:=\operatorname{load}(e)): i s)=\phi(\rho[x \mapsto \perp], i s) \\
& \phi(\rho,(x:=\operatorname{protect}(e)): i s)=\phi(\rho[x \mapsto \perp], \text { is }) \\
& \phi(\rho, i: i s)=\phi(\rho, i s)
\end{aligned}
$$

(a) Transient variable map.

$$
\begin{aligned}
& \text { EXECUTE } \\
& \frac{\left|i s_{1}\right|=n-1 \quad \rho^{\prime}=\phi\left(i s_{1}, \rho\right) \quad\left\langle i s_{1}, i, i s_{2}, c s\right\rangle \stackrel{\left(\mu, \rho^{\prime}, o\right)}{\longrightarrow}\left\langle i s^{\prime}, c s^{\prime}\right\rangle}{\left\langle i s_{1}+[i]+i s_{2}, c s, \mu, \rho\right\rangle \stackrel{\operatorname{exec} n}{\longrightarrow}\left\langle i s^{\prime}, c s^{\prime}, \mu, \rho\right\rangle}
\end{aligned}
$$

(b) Execute rule.

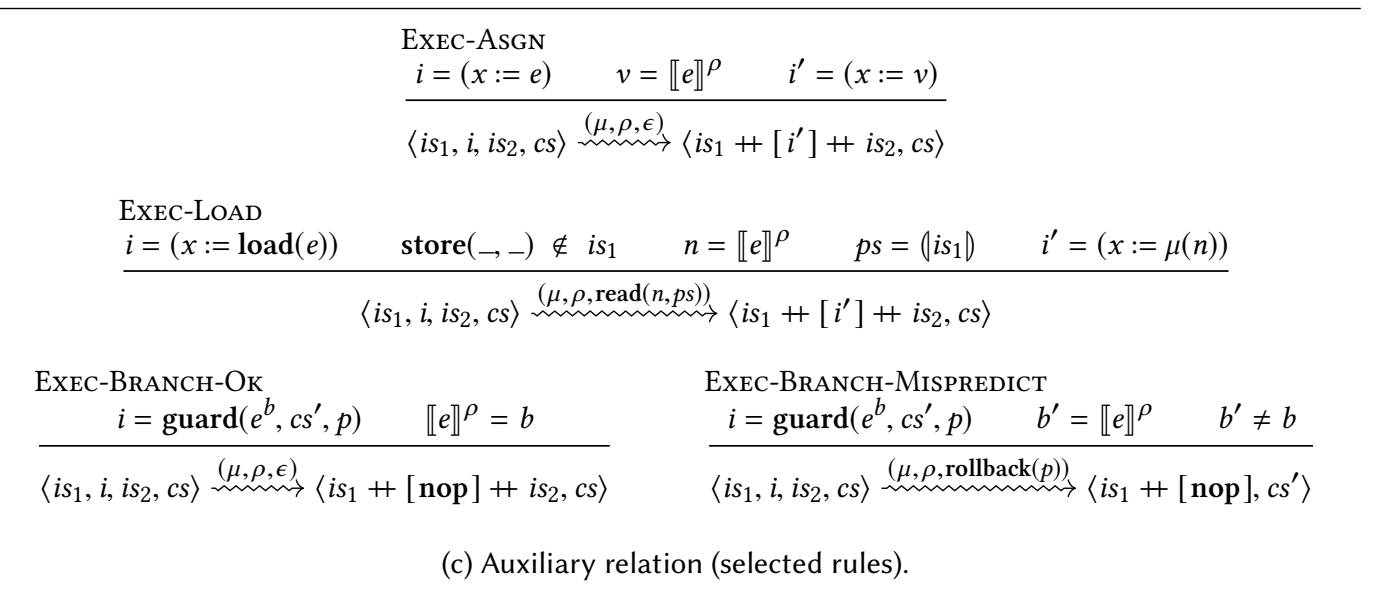

Fig. 6. Execute stage.

\subsection{Execute Stage}

In the execute stage, the processor evaluates the operands of instructions in the reorder buffer and rolls back the program state whenever it detects a misprediction.

Transient Variable Map. Since instructions can be executed out-of-order, when we evaluate operands we need to take into account how previous, possibly unresolved assignments in the reorder buffer affect the variable map. In particular, we need to ensure that an instruction cannot execute if it depends on a preceding assignment whose value is still unknown. To this end, we define a function $\phi(\rho$, is), called the transient variable map (Fig. 6a), which updates variable map $\rho$ with the pending assignments in reorder buffer $i$. The function walks through the reorder buffer, registers each resolved assignment instruction $(x:=v)$ in the variable map through function update $\rho[x \mapsto v]$, and marks variables from pending assignments (i.e., $x:=e, x:=\operatorname{load}(e)$, and $x:=\operatorname{protect}(r))$ as undefined $(\rho[x \mapsto \perp])$, making their respective values unavailable to following instructions. 
Execute Rule and Auxiliary Relation. Figure 6 shows selected rules for the execute stage. Rule [EXECUTE] executes the $n$th instruction in the reorder buffer, following the directive exec $n$. For this, the rule splits the reorder buffer into prefix $i s_{1}, n$th instruction $i$, and suffix $i s_{2}$. Next, it computes the transient variable map $\phi\left(i s_{1}, \rho\right)$ and executes a transition step under the new map using an auxiliary relation $\rightsquigarrow$. Notice that [EXECUTE] does not update the store or the variable map-the transient map is simply discarded. These changes are performed later in the retire stage.

The rules for the auxiliary relation are shown in Fig. 6c. The relation transforms a tuple $\left\langle i s_{1}, i, i s_{2}, c s\right\rangle$ consisting of prefix, suffix and current instruction $i$ into a tuple $\left\langle i s^{\prime}, c s^{\prime}\right\rangle$ specifying the reorder buffer and command stack obtained by executing $i$. For example, rule [ExEC-AsGN] evaluates the right-hand side of the assignment $x:=e$ where $\llbracket e \rrbracket^{\rho}$ denotes the value of $e$ under $\rho$. The premise $v=\llbracket e \rrbracket^{\rho}$ ensures that the expression is defined, i.e., it does not evaluate to $\perp$. Then, the rule substitutes the computed value into the assignment $(x:=v)$, and reinserts the instruction back into its original position in the reorder buffer.

Loads. Rule [ExEC-LOAD] executes a memory load. The rule computes the address $\left(n=\llbracket e \rrbracket^{\rho}\right)$, retrieves the value at that address from memory $(\mu(n))$ and rewrites the load into an assignment $(x:=\mu(n))$. By inserting the resolved assignment into the reorder buffer, the rule allows the processor to transiently forward the loaded value to later instructions through the transient variable map. To record that the load is issued speculatively, the observation $\operatorname{read}(n, p s)$ stores list $p s$ containing the identifiers of the guard and fail instructions still pending in the reorder buffer. Function $\left(i s_{1}\right)$ simply extracts these identifiers from the guard and fail instructions in prefix $i s_{1}$. In the rule, premise store $\left({ }_{-},\right)_{i} \notin i s_{1}$ prevents the processor from reading potentially stale data from memory: if the load aliases with a preceding (but pending) store, ignoring the store could produce a stale read.

Store Forwarding. Alternatively, instead of exclusively reading fresh values from memory, it would be also possible to forward values waiting to be written to memory at the same address by a pending, aliasing store. For example, if buffer $i s_{1}$ contained a resolved instruction store $(n, v)$, rule [ExECLOAD] could directly propagate the value $v$ to the load, which would be resolved to $x:=v$, without reading memory and thus generating silent event $\epsilon$ instead of $\operatorname{read}(n, p s) .{ }^{5}$ The Spectre v1.1 variant relies on this particular microarchitectural optimization to leak data-for example by speculatively writing transient data out of the bounds of an array, forwarding that data to an aliasing load.

Guards and Rollback. Rules [EXEC-BRANCH-OK] and [EXEC-BRANCH-MISPREDICT] resolve guard instructions. In rule [EXEC-BRANCH-OK], the predicted and computed value of the guard expression match $\left(\llbracket e \rrbracket^{\rho}=b\right)$, and thus the processor only replaces the guard with nop. In contrast, in rule [EXEC-BRANCH-MisPREDICT] the predicted and computed value differ $\left(\llbracket e \rrbracket^{\rho}=b^{\prime}\right.$ and $\left.b^{\prime} \neq b\right)$. This causes the processor to revert the program state and issue a rollback observation (rollback $(p))$. For the rollback, the processor discards the instructions past the guard (i.e., $i s_{2}$ ) and substitutes the current commands stack $c s$ with the rollback stack $c s^{\prime}$ which causes execution to revert to the alternative branch.

\subsection{Retire Stage}

The retire stage removes completed instructions from the reorder buffer and propagates their changes to the variable map and memory store. While instructions are executed out-of-order, they are retired in-order to preserve the illusion of sequential execution to the user. For this reason, the rules for the retire stage in Figure 7 always remove the first instruction in the reorder buffer. For example, rule [RetiRE-Nop] removes nop from the front of the reorder buffer. Rules [RETIRE-AsGn]

\footnotetext{
${ }^{5}$ Capturing the semantics of store-forwarding would additionally require some bookkeeping about the freshness of (forwarded) values in order to detect reading stale data. We refer the interested reader to [Cauligi et al. 2020] for full details.
} 
RETIRE-NOP

$$
\langle\text { nop : is, cs, } \mu, \rho\rangle \stackrel{\text { retire }}{\longrightarrow}_{\epsilon}\langle i s, c s, \mu, \rho\rangle
$$

RETIRE-STORE

$\langle\operatorname{store}(n, v): i s, c s, \mu, \rho\rangle \stackrel{\text { retire }}{\longrightarrow}_{\epsilon}\langle i s, c s, \mu[n \mapsto v], \rho\rangle$

\section{RETIRE-AsGN}

$\langle x:=v: i s, c s, \mu, \rho\rangle \stackrel{\text { retire }}{\longrightarrow}_{\epsilon}\langle i s, c s, \mu, \rho[x \mapsto v]\rangle$

RETIRE-FAIL

Fig. 7. Retire stage.

\begin{tabular}{|c|c|}
\hline Memory & Layou \\
\hline$\mu(0)=0$ & $b[0]$ \\
\hline$\mu(1)=0$ & $a[0]$ \\
\hline$\mu(2)=0$ & $a[1]$ \\
\hline$\mu(3)=42$ & $s[0]$ \\
\hline$\ldots$ & \\
\hline
\end{tabular}

$$
\begin{gathered}
\text { Variable Map } \\
\rho\left(i_{1}\right)=1 \\
\rho\left(i_{2}\right)=2 \\
\ldots
\end{gathered}
$$

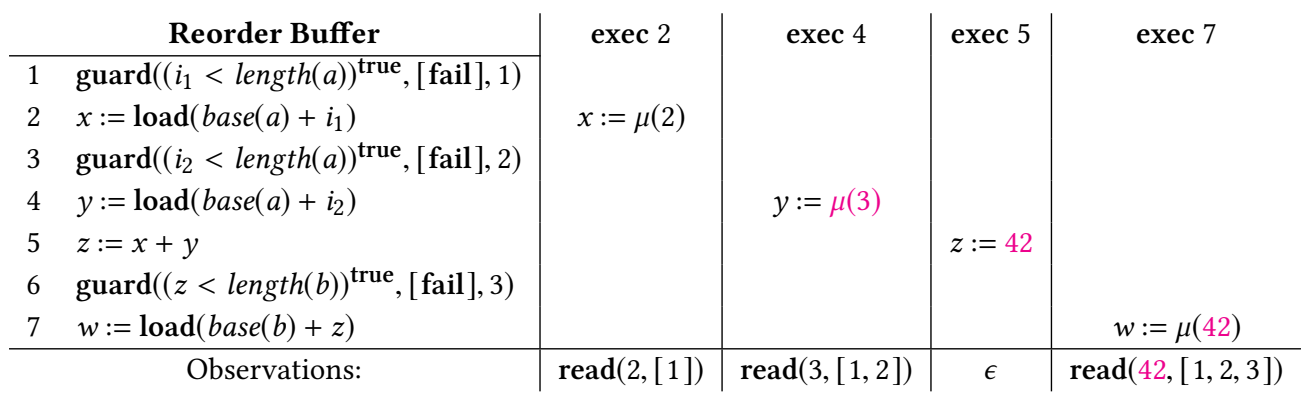

Fig. 8. Leaking execution of running program ExAMPLE.

and [RetiRe-Store] remove the resolved assignment $x:=v$ and instruction store $(n, v)$ from the reorder buffer and update the variable map $(\rho[x \mapsto v])$ and the memory store $(\mu[n \mapsto v])$ respectively. Rule [RETIRE-FAIL] aborts the program by emptying reorder buffer and command stack and generates a fail $(p)$ observation, simulating a processor raising an exception (e.g., a segmentation fault).

Example. We demonstrate how the attacker can leak a secret from program ExAMPLE (Fig. 2) in our model. First, the attacker instructs the processor to fetch all the instructions, supplying prediction true for all bounds-check conditions. Figure 8 shows the resulting buffer and how it evolves after each attacker directive; the memory $\mu$ and variable map $\rho$ are shown above. The attacker directives instruct the processor to speculatively execute the load instructions and the assignment (but not the guard instructions). Directive exec 2 executes the first load instruction by computing the memory address $2=\llbracket$ base $(a)+i_{1} \rrbracket^{\rho}$ and replacing the instruction with the assignment $x:=\mu(2)$ containing the loaded value. Directive exec 4 transiently reads public array $a$ past its bound, at index 2 , reading into the memory $(\mu(3)=42)$ of secret array $s[0]$ and generates the corresponding observation. Finally, the processor forwards the values of $x$ and $y$ through the transient variable map $\rho[x \mapsto \mu(2), y \mapsto \mu(3)]$ to compute their sum in the fifth instruction, $(z:=42)$, which is then used as an index in the last instruction and leaked to the attacker via observation $\operatorname{read}(42,[1,2,3])$.

\subsection{Protect}

Next, we turn to the rules that formalize the semantics of protect as an ideal hardware primitive and then its software implementation via speculative-load-hardening (SLH). 


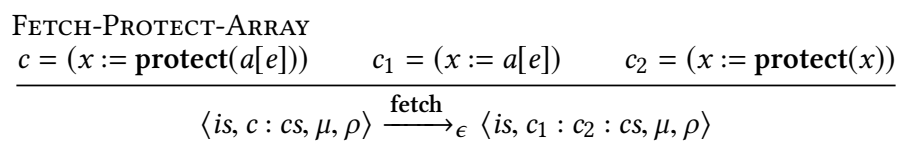

Fetch-Protect-Expr

$$
\frac{c=(x:=\operatorname{protect}(e)) \quad i=(x:=\operatorname{protect}(e))}{\langle i s, c: c s, \mu, \rho\rangle \stackrel{\text { fetch }}{\longrightarrow}_{\epsilon}\langle i s+[i], c s, \mu, \rho\rangle}
$$

$$
\frac{\operatorname{Exec}_{i=(x)=\operatorname{protect}} 1}{\left.\left\langle i s_{1}, i, i s_{2}, c s\right\rangle\right) \stackrel{(\mu, \rho, \epsilon)}{m}\left\langle i s_{1}+\left[i^{\prime}\right]+i s_{2}, c s\right\rangle}
$$

$$
\begin{aligned}
& \text { Exec-Protect } 2 \\
& \frac{i=(x:=\operatorname{protect}(v)) \quad \operatorname{guard}\left({ }_{-},+-\right) \notin i s_{1} \quad i^{\prime}=(x:=v)}{\left\langle i s_{1}, i, i s_{2}, c s\right\rangle \stackrel{(\mu, \rho, \epsilon)}{\sim}\left\langle i s_{1}+\left[i^{\prime}\right]+i s_{2}, c s\right\rangle}
\end{aligned}
$$

(a) Semantics of protect as a hardware primitive (selected rules).

$$
\begin{aligned}
& \text { Fetch-Protect-SLH } \\
& c=x:=\operatorname{protect}(a[e]) \quad e_{1}=e<\operatorname{length}(a) \quad e_{2}=\operatorname{base}(a)+e \\
& c_{1}=m:=e_{1} \quad c_{2}=m:=m ? \mathbf{1 : 0} \quad c_{3}=x:=*\left(e_{2} \otimes m\right) \quad c^{\prime}=c_{1} ; \text { if } m \text { then } c_{2} ; c_{3} \text { else fail } \\
& \langle i s, c: c s, \mu, \rho\rangle \stackrel{\text { fetch }}{\longrightarrow}\left\langle i s, c^{\prime}: c s, \mu, \rho\right\rangle
\end{aligned}
$$

(b) Software implementation of $\operatorname{protect}(a[e])$.

Fig. 9. Semantics of protect.

Protect in Hardware. Instruction $x:=\operatorname{protect}(r)$ assigns the value of $r$, only after all previous guard instructions have been executed, i.e., when the value has become stable and no more rollbacks are possible. Figure 9a formalizes this intuition. Rule [Fетсн-Рвотест-ExPR] fetches protect commands involving simple expressions $(x:=\operatorname{protect}(e))$ and inserts the corresponding protect instruction in the reorder buffer. Rule [Fетсн-Рвотест-ARRAY] piggy-backs on the previous rule by splitting a protect of an array read $(x:=\operatorname{protect}(a[e]))$ into a separate assignment of the array value $(x:=a[e])$ and protect of the variable $(x:=\operatorname{protect}(x))$. Rules [Exec-Protect $\left.{ }_{1}\right]$ and [Exec-Protect ${ }_{2}$ ] extend the auxiliary relation $\rightsquigarrow$. Rule [ExEc-PRотест ${ }_{1}$ ] evaluates the expression $\left(v=\llbracket e \rrbracket^{\rho}\right)$ and reinserts the instruction in the reorder buffer as if it were a normal assignment. However, the processor leaves the value wrapped inside the protect instruction in the reorder buffer, i.e., $x:=\operatorname{protect}(v)$, to prevent forwarding the value to the later instructions via the the transient variable map. When no guards are pending in the reorder buffer $\left(\operatorname{guard}(-,-,-) \notin i s_{1}\right)$, rule [Exec-PROTECT ${ }_{2}$ ] transforms the instruction into a normal assignment, so that the processor can propagate and commit its value.

Example. Consider again EXAMPLE and the execution shown in Figure 8. In the repaired program, $x+y$ is wrapped in a protect statement. As a result, directive exec 5 produces value $z:=\operatorname{protect}(42)$, instead of $z:=42$ which prevents instruction 7 from executing (as its target address is undefined), until all guards are resolved. This in turn prevents leaking the transient value.

Protect in Software. The software implementation of protect applies SLH to array reads. Intuitively, we rewrite array reads by injecting artificial data-dependencies between bounds-check conditions 
and the corresponding addresses in load instructions, thus transforming control-flow dependencies into data-flow dependencies. ${ }^{6}$ These data-dependencies validate control-flow decisions at runtime by stalling speculative loads until the processor resolves their bounds check conditions. ${ }^{7}$ Formally, we replace rule [Fетсh-Protect-Array] with rule [Fетсh-Рrotect-SLH] in Figure 9b. The rule computes the bounds check condition $e_{1}=e<\operatorname{length}(a)$, the target address $e_{2}=$ base $(a)+e$, and generates commands that abort the execution if the check fails, like for regular array reads. Additionally, the rule generates regular commands that (i) assign the result of the bounds check to a reserved variable $m\left(c_{1}=m:=e_{1}\right)$, (ii) conditionally update the variable with a bitmask consisting of all $1 \mathrm{~s}$ or $0 \mathrm{~s}\left(c_{2}=m:=m ? \mathbf{1}: \mathbf{0}\right)$, and (iii) mask off the target address with the bitmask $\left(c_{3}=x:=*\left(e_{2} \otimes m\right)\right) .{ }^{8}$ Since the target address in command $c_{3}$ depends on variable $m$, the processor cannot read memory until the bounds check is resolved. If the check succeeds, the bitmask $m=1$ leaves the target address unchanged $\left(\llbracket e^{\prime} \rrbracket^{\rho}=\llbracket e^{\prime} \otimes 1 \rrbracket^{\rho}\right)$ and the processor reads the correct address normally. Otherwise, the bitmask $m=0$ zeros out the target address and the processor loads speculatively only from the constant address $0=\llbracket e^{\prime} \otimes 0 \rrbracket^{\rho}$. (We assume that the processor reserves the first memory cell and initializes it with a dummy value, e.g., $\mu(0)=0$.) Notice that this solution works under the assumption that the processor does not evaluate the conditional update $m:=m$ ? $\mathbf{1}: \mathbf{0}$ speculatively. We can easily enforce that by compiling conditional updates to non-speculative instructions available on commodity processors (e.g., the conditional move instruction CMOV on x86).

Example. Consider again EXAMPLE. The optimal patch protect $(x+y)$ cannot be executed on existing processors without support for a generic protect primitive. Nevertheless, we can repair the program by applying SLH to the individual array reads, i.e., $x:=\operatorname{protect}\left(a\left[i_{1}\right]\right)$ and $y:=\operatorname{protect}\left(a\left[i_{2}\right]\right)$.

\section{TYPE SYSTEM AND INFERENCE}

In Section 4.1, we present a transient-flow type system which statically rejects programs that can potentially leak through transient execution attacks. These speculative leaks arise in programs as transient data flows from source to sink expressions. Our type system does not rely on user annotations to identify secret. Indeed, our typing rules simply ignore security annotations and instead, conservatively reject programs that exhibit any source-to-sink data flows. Intuitively, this is because security annotations are not trustworthy when programs are executed speculatively: public variables could contain transient secrets and secret variables could flow speculatively into public sinks. Additionally, our soundness theorem, which states that well-typed programs are speculatively constant-time, assumes that programs are sequentially constant-time. We do not enforce a sequential constant-time discipline; this can be done using existing constant-time type systems or interfaces (e.g., [Watt et al. 2019; Zinzindohoué et al. 2017]).

Given an unannotated program, we apply constraint-based type inference [Aiken 1996; Nielson and Nielson 1998] to generate its use-def graph and reconstruct type information (Section 4.2). Then, reusing off-the-shelf Max-Flow/Min-Cut algorithms, we analyze the graph and locate potential speculative vulnerabilities in the form of a variable min-cut set. Finally, using a simple program

\footnotetext{
${ }^{6}$ Technically, applying SLH to array reads is a program transformation. In our implementation (§ 6), the SLH version of protect inserts additional instructions into the program to perform the conditional update and mask operation described in rule [Fетсн-Protect-SLH].

${ }^{7}$ A fully-fledged security tool could apply static analysis techniques to infer array bounds. Our implementation of BLADE merely simulates SLH using a static constant instead of the actual lengths, as discussed in Section 6.

${ }^{8}$ Alternatively, it would be also possible to mask the loaded value, i.e., $c_{3}=x:=\left(* e_{2}\right) \otimes m$. However, this alternative mitigation would still introduce transient data in various processor internal buffers, where it could be leaked. In contrast, we conservatively mask the address, which has the effect of stalling the load and preventing transient data from even entering the processor, thus avoiding the risk of leaking it altogether.
}

Proc. ACM Program. Lang., Vol. 5, No. POPL, Article 49. Publication date: January 2021. 


$$
\begin{aligned}
& \text { VAlue } \\
& \Gamma \vdash v: \tau \Rightarrow \emptyset
\end{aligned} \quad \frac{\Gamma(x)=\tau}{\Gamma \vdash x: \tau \Rightarrow x \sqsubseteq \alpha_{x}}
$$

$$
\begin{aligned}
& \text { Bop } \\
& \begin{array}{c}
\Gamma \vdash e_{1}: \tau_{1} \Rightarrow k_{1} \quad \Gamma \vdash e_{2}: \tau_{2} \Rightarrow k_{2} \quad \tau_{1} \sqsubseteq \tau \quad \tau_{2} \sqsubseteq \tau \\
\Gamma \vdash e_{1} \oplus e_{2}: \tau \Rightarrow k_{1} \cup k_{2} \cup\left(e_{1} \sqsubseteq e_{1} \oplus e_{2}\right) \cup\left(e_{2} \sqsubseteq e_{1} \oplus e_{2}\right)
\end{array} \\
& \text { ARRAY-READ } \\
& \frac{\Gamma \vdash e: S \Rightarrow k}{\Gamma \vdash a[e]: \mathrm{T} \Rightarrow k \cup(e \sqsubseteq \mathrm{S}) \cup(\mathrm{T} \sqsubseteq a[e])}
\end{aligned}
$$

(a) Typing rules for expressions and arrays.

$$
\begin{aligned}
& \text { AsGn } \\
& \Gamma \vdash r: \tau \Rightarrow k \quad \tau \sqsubseteq \Gamma(x) \\
& \hline, \operatorname{Prot} \vdash x:=r \Rightarrow k \cup(r \sqsubseteq x)
\end{aligned}
$$

\section{Asgn-Prot}

$$
\frac{\Gamma \vdash r: \tau \Rightarrow k \quad x \in \operatorname{Prot}}{\Gamma, \operatorname{Prot} \vdash x:=r \Rightarrow k \cup(r \sqsubseteq x)}
$$

Protect

$$
\frac{\Gamma \vdash r: \tau \Rightarrow k}{\Gamma, \operatorname{Prot} \vdash x:=\operatorname{protect}(r) \Rightarrow k}
$$

$$
\begin{aligned}
& \text { IF-THEN-ELSE } \\
& \Gamma \vdash e: \mathrm{S} \Rightarrow k \quad \Gamma \text {, Prot } \vdash c_{1} \Rightarrow k_{1} \quad \Gamma \text {, Prot } \vdash c_{2} \Rightarrow k_{2} \\
& \Gamma \text {, Prot } \vdash \text { if } e \text { then } c_{1} \text { else } c_{2} \Rightarrow k \cup k_{1} \cup k_{2} \cup(e \sqsubseteq \mathrm{S})
\end{aligned}
$$

(b) Typing rules for commands.

Fig. 10. Transient-flow type system and constraints generation .

repair algorithm we patch the program by inserting a minimum number of protect so that it cannot leak speculatively anymore (Section 4.3).

\subsection{Type System}

Our type system assigns a transient-flow type to expressions and tracks how transient values propagate within programs, rejecting programs in which transient values reach commands which may leak them. An expression can either be typed as stable (S) indicating that it cannot contain transient values during execution, or as transient $(\mathrm{T})$ indicating that it can. These types form a 2-point lattice [Landauer and Redmond 1993], which allows stable expressions to be typed as transient, but not vice versa, i.e., we define a can-flow-to relation $\sqsubseteq$ such that $\mathrm{S} \sqsubseteq \mathrm{T}$, but $\mathrm{T} \nsubseteq \mathrm{S}$.

Typing Expressions. Given a typing environment for variables $\Gamma \in \operatorname{Var} \rightarrow\{\mathrm{S}, \mathrm{T}\}$, the typing judgment $\Gamma \vdash r: \tau$ assigns a transient-flow type $\tau$ to $r$. Figure 10 presents selected rules (see [Vassena et al. 2020] for the rest). The shaded part of the rules generates type constraints during type inference and are explained later. Values can assume any type in rule [VALUE] and variables are assigned their respective type from the environment in rule [VAR]. Rule [Bop] propagates the 
type of the operands to the result of binary operators $\oplus \in\{+,<, \otimes\}$. Finally, rule [ARRAY-READ] assigns the transient type $\mathrm{T}$ to array reads as the array may potentially be indexed out of bounds during speculation. Importantly, the rule requires the index expression to be typed stable (S) to prevent programs from leaking through the cache.

Typing Commands. Given a set of implicitly protected variables Prot, we define a typing judgment $\Gamma$, Prot $\vdash c$ for commands. Intuitively, a command $c$ is well-typed under environment $\Gamma$ and set Prot, if $c$ does not leak, under the assumption that the expressions assigned to all variables in Prot are protected using the protect primitive. Figure 10b shows our typing rules. Rule [AsGN] disallows assignments from transient to stable variables (as T $\$$ S). Rule [Рвотест] relaxes this policy as long as the right-hand side is explicitly protected. ${ }^{9}$ Intuitively, the result of protect is stable and it can thus flow securely to variables of any type. Rule [AsGN-Prot] is similar, but instead of requiring an explicit protect statement, it demands that the variable is accounted for in the protected set Prot. This is secure because all assignments to variables in Prot will eventually be protected through the repair function discussed later in this section. Rule [ARRAY-WRITE] requires the index expression used in array writes to be typed stable (S) to avoid leaking through the cache, similarly to rule [ARRAY-READ] Notice that the rule does not prevent storing transient data, i.e., the stored value can have any type While this is sufficient to mitigate Spectre v1 attacks, it is inadequate to defend against Spectre v1.1. Intuitively, the store-to-load forward optimization enables additional implicit data-flows between stored data to aliasing load instructions, thus enabling Spectre v1.1 attacks. Luckily, to protect against these attacks we need only modify one clause of rule [ARRAY-WRITE]: we change the type system to conservatively treat stored values as sinks and therefore require them to be typed stable $\left(\Gamma \vdash e_{2}: S\right) .{ }^{10}$

Implicit Flows. To prevent programs from leaking data implicitly through their control flow, rule [IF-THEN-ELSE] requires the branch condition to be stable. This might seem overly restrictive, at first: why can't we accept a program that branches on transient data, as long as it does not perform any attacker-observable operations (e.g., memory reads and writes) along the branches? Indeed, classic information-flow control (IFC) type systems (e.g., [Volpano et al. 1996]) take this approach by keeping track of an explicit program counter label. Unfortunately, such permissiveness is unsound under speculation. Even if a branch does not contain observable behavior, the value of the branch condition can be leaked by the instructions that follow a mispredicted branch. In particular, upon a rollback, the processor may repeat some load and store instructions after the mispredicted branch and thus generate additional observations, which can implicitly reveal the value of the branch condition.

Example. Consider the program $\{$ if $\operatorname{tr}$ then $x:=0$ else skip $\} ; y:=a[0]$. The program can leak the transient value of $t r$ during speculative execution. To see that, assume that the processor predicts that $\operatorname{tr}$ will evaluate to true. Then, the processor speculatively executes the then-branch $(x:=0)$ and the load instruction $(y:=a[0])$, before resolving the condition. If $t r$ is true, the observation trace of the program contains a single read observation. However, if $t r$ is false, the processor detects a misprediction, restarts the execution from the other branch (skip) and executes the array read again, producing a rollback and two read observations. From these observations, an attacker could potentially make inferences about the value of $t r$. Consequently, if $t r$ is typed as $\mathrm{T}$, our type system rejects the program as unsafe.

\subsection{Type Inference}

Our type-inference approach is based on type-constraints satisfaction [Aiken 1996; Nielson and Nielson 1998]. Intuitively, type constraints restrict the types that variables and expressions may

\footnotetext{
${ }^{9}$ Readers familiar with information-flow control may see an analogy between protect and the declassify primitive of some IFC languages [Myers et al. 2004].

${ }^{10}$ Both variants of rule [ARRAY-WrITE] are implemented in BLADE (§ 6) and evaluated (§ 7).
} 
assume in a program. In the constraints, the possible types of variables and expressions are represented by atoms consisting of unknown types of expressions and variables. Solving these constrains requires finding a substitution, i.e., a mapping from atoms to concrete transient-flow types, such that all constraints are satisfied if we instantiate the atoms with their type.

Our type inference algorithm consists of 3 steps: (i) generate a set of type constraints under an initial typing environment and protected set that under-approximates the solution of the constraints, (ii) construct the def-use graph from the constraints and find a cut-set, and (iii) cut the transient-tostable dataflows in the graph and compute the resulting typing environment. We start by describing the generation of constraints through the typing judgment from Figure 10.

Type Constraints. Given a typing environment $\Gamma$, a protected set Prot, the judgment $\Gamma$, Prot $\vdash r \Rightarrow$ $k$ type checks $r$ and generates type constraints $k$. The syntax for constraints is shown in Figure 11. Constraints are sets of can-flow-to relations involving concrete types ( $\mathrm{S}$ and $\mathrm{T}$ ) and atoms, i.e., type variables corresponding to program variables (e.g., $\alpha_{x}$ for $x$ ) and unknown types for expressions (e.g., $r$ ). In rule [VAR], constraint $x \sqsubseteq \alpha_{x}$ indicates that the type variable of $x$ should be at least as transient as the unknown type of $x$. This ensures that, if variable $x$ is transient, then $\alpha_{x}$ can only be instantiated with type T. Rule [Bop] generates constraints $e_{1} \sqsubseteq e_{1} \oplus e_{2}$ and $e_{2} \sqsubseteq e_{1} \oplus e_{2}$ to reflect the fact that the unknown type of $e_{1} \oplus e_{2}$ should be at least as transient as the (unknown) type of $e_{1}$ and $e_{2}$. Notice that these constraints correspond exactly to the premises $\tau_{1} \sqsubseteq \tau$ and $\tau_{2} \sqsubseteq \tau$ of the same rule. Similarly, rule [ARRAY-READ] generates constraint $e \sqsubseteq \mathrm{S}$ for the unknown type of the array index, thus forcing it to be typed $S .{ }^{11}$ In addition to these, the rule generates also the constraint $\mathrm{T} \sqsubseteq a[e]$, which forces the type of $a[e]$ to be T. Rule [AsGN] generates the constraint $r \sqsubseteq x$ disallowing transient to stable assignments. In contrast, rule [РRотест] does not generate the constraint $r \sqsubseteq x$ because $r$ is explicitly protected. Rule [AsGN-PROT] generates the same constraint as rule [AsGN], because type inference ignores the protected set, which is computed in the next step of the algorithm. The constraints generated by the other rules follow the same intuition. In the following we describe the inference algorithm in more detail.

Generating Constraints. We start by collecting a set of constraints $k$ via typing judgement $\Gamma$, Prot $\vdash$ $s \Rightarrow k$. For this, we define a dummy environment $\Gamma^{*}$ and protected set Prot $^{*}$, such that $\Gamma^{*}$, Prot ${ }^{*}$ $c \Rightarrow k$ holds for any command $c$, (i.e., we let $\Gamma^{*}=\lambda x . S$ and include all variables in the cut-set) and use it to extract the set of constraints $k$.

Solutions and Satisfiability. We define the solution to a set of constraints as a function $\sigma$ from atoms to flow types, i.e., $\sigma \in$ AтомS $\mapsto\{\mathrm{T}, \mathrm{S}\}$, and extend solutions to map $\mathrm{T}$ and $\mathrm{S}$ to themselves. For a set of constraints $k$ and a solution function $\sigma$, we write $\sigma \vdash k$ to say that the constraints $k$ are satisfied under solution $\sigma$. A solution $\sigma$ satisfies $k$, if all can-flow-to constraints hold, when the atoms are replaced by their values under $\sigma$ (Fig. 11). We say that a set of constraints $k$ is satisfiable, if there is a solution $\sigma$ such that $\sigma \vdash k$.

Def-Use Graph \& Paths. The constraints generated by our type system give rise to the def-use graph of the type-checked program. For a set of constraints $k$, we call a sequence of atoms $a_{1} \ldots a_{n}$ a path in $k$, if $a_{i} \sqsubseteq a_{i+1} \in k$ for $i \in\{1, \ldots, n-1\}$ and say that $a_{1}$ is the path's entry and $a_{n}$ its exit. A T-S path is a path with entry T and exit S. A set of constraints $k$ is satisfiable if and only if there is no T-S path in $k$, as such a path would correspond to a derivation of false. If $k$ is satisfiable, we can compute a solution $\sigma(k)$ by letting $\sigma(k)(a)=\mathrm{T}$, if there is a path with entry $\mathrm{T}$ and exit $a$, and $\mathrm{S}$ otherwise.

Cuts. If a set of constraints is unsatisfiable, we can make it satisfiable by removing some of the nodes in its graph or equivalently protecting some of the variables. A set of atoms A cuts a path

\footnotetext{
${ }^{11}$ No constraints are generated for the type of the array because our syntax forces the array to be a static, constant value. If we allowed arbitrary expressions for arrays the rule would require them to by typed as stable.
} 


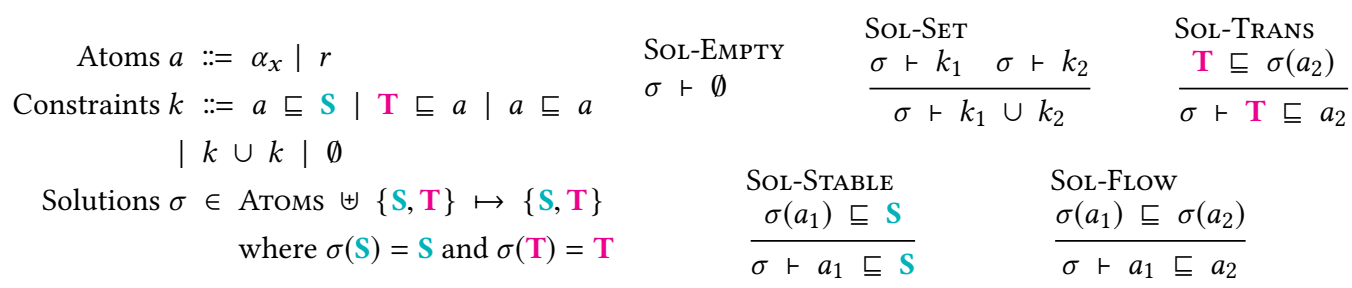

Fig. 11. Type constraints and satisfiability.

$a_{1} \ldots a_{n}$, if some $a \in A$ occurs along the path, i.e., there exists $a \in A$ and $i \in\{1, \ldots, n\}$ such that $a_{i}=a$. We call $A$ a cut-set for a set of constraints $k$, if $A$ cuts all T-S paths in $k$. A cut-set $A$ is minimal for $k$, if all other cut-sets $A^{\prime}$ contain as many or more atoms than $A$, i.e., $\# A \leqslant \# A^{\prime}$.

Extracting Types From Cuts. From a set of variables $A$ such that $A$ is a cut-set of constraints $k$, we can extract a typing environment $\Gamma(k, A)$ as follows: for an atom $\alpha_{x}$, we define $\Gamma(k, A)(x)=\mathrm{T}$, if there is a path with entry $\mathrm{T}$ and exit $\alpha_{x}$ in $k$ that is not cut by $A$, and let $\Gamma(k, A)(x)=\mathrm{S}$ otherwise.

Proposition 1 (Type Inference). If $\Gamma^{*}$, Prot ${ }^{*} \vdash c \Rightarrow k$ and $A$ is a set of variables that cut $k$, then $\Gamma(k, A), A \vdash c$.

Remark. To infer a repair using exclusively SLH-based protect statements, we simply restrict our cut-set to only include variables that are assigned from an array read.

Example. Consider again EXAMPLE in Figure 2. The graph defined by the constraints $k$, given by $\Gamma^{*}$, Prot ${ }^{*}+$ ExAmple $\Rightarrow k$ is shown in Figure 3, where we have omitted $\alpha$-nodes. The constraints are not satisfiable, since there are T-S paths. Both $\{x, y\}$ and $\{z\}$ are cut-sets, since they cut each T-S path, however, the set $\{z\}$ contains only one element and is therefore minimal. The typing environment $\Gamma(k,\{x, y\})$ extracted from the sub-obptimal cut $\{x, y\}$ types all variables as $\mathrm{S}$, while the typing extracted from the optimal cut, i.e., $\Gamma(k,\{z\})$ types $x$ and $y$ as $\mathrm{T}$ and $z, i_{1}$ and $i_{2}$ as S. By Proposition 1 both $\Gamma(k,\{x, y\}),\{x, y\} \vdash$ EXAMPLE and $\Gamma(k,\{z\}),\{z\} \vdash$ EXAMPLE hold.

\subsection{Program Repair}

As a final step, our repair algorithm repair(c, Prot) traverses program $c$ and inserts a protect statement for each variable in the cut-set Prot. For simplicity, we assume that programs are in static single assignment (SSA) form. ${ }^{12}$ Therefore, for each variable $x \in$ Prot there is a single assignment $x:=r$, and our repair algorithm simply replaces it with $x:=\operatorname{protect}(r)$.

\section{CONSISTENCY AND SECURITY}

We now present two formal results about our speculative semantics and the security of our type system. First, we prove that the semantics from Section 3 is consistent with sequential program execution (Theorem 1). Intuitively, programs running on our processor produce the same results (with respect to the memory store and variables) as if their commands were executed in-order and without speculation. The second result establishes that our type system is sound (Theorem 2). We prove that our transient-flow type system in combination with a standard constant-time type system (e.g., [Protzenko et al. 2019; Watt et al. 2019]) enforces constant time under speculative

\footnotetext{
${ }^{12}$ We make this assumption to simplify our analysis and security proof. We also omit phi-nodes from our calculus to avoid cluttering the semantics and remark that this simplification does not affect our flow-insensitive analysis. Our implementation operates on Cranelift's SSA form (§6).
} 
execution [Cauligi et al. 2020]. We provide full definitions and proofs in the extended version of this paper [Vassena et al. 2020].

Consistency. We write $C \Downarrow_{O}^{D} C^{\prime}$ for the complete speculative execution of configuration $C$ to final configuration $C^{\prime}$, which generates a trace of observations $O$ under list of directives $D$. Similarly, we write $\langle\mu, \rho\rangle \Downarrow_{O}^{c}\left\langle\mu^{\prime}, \rho^{\prime}\right\rangle$ for the sequential execution of program $c$ with initial memory $\mu$ and variable map $\rho$ resulting in final memory $\mu^{\prime}$ and variable map $\rho^{\prime}$. To relate speculative and sequential observations, we define a projection function, written $O \downarrow$, which removes prediction identifiers, rollbacks, and misspeculated loads and stores.

Theorem 1 (Consistency). For all programs $c$, initial memory stores $\mu$, variable maps $\rho$, and directives $D$, if $\langle\mu, \rho\rangle \Downarrow_{O}^{c}\left\langle\mu^{\prime}, \rho^{\prime}\right\rangle$ and $\langle[],[c], \mu, \rho\rangle \Downarrow_{O^{\prime}}^{D}\left\langle[],[], \mu^{\prime \prime}, \rho^{\prime \prime}\right\rangle$, then $\mu^{\prime}=\mu^{\prime \prime}, \rho^{\prime}=\rho^{\prime \prime}$, and $O \cong O^{\prime} \downarrow$.

The theorem ensures equivalence of the final memory stores, variable maps, and observation traces from the sequential and the speculative execution. Notice that trace equivalence is up to permutation, i.e., $O \cong O^{\prime} \downarrow$, because the processor can execute load and store instructions out-oforder.

Speculative Constant Time. In our model, an attacker can leak information through the architectural state (i.e., the variable map and the memory store) and through the cache by supplying directives that force the execution of an otherwise constant-time cryptographic program to generate different traces. In the following, the relation $\approx_{L}$ denotes $L$-equivalence, i.e., equivalence of configurations with respect to a security policy $L$ that specifies which variables and arrays are public $(L)$ and attacker observable. Initial and final configurations are $L$-equivalent $\left(C_{1} \approx_{L} C_{2}\right)$ if the values of public variables in the variable maps and the content of public arrays in the memories coincide, i.e., $\forall x \in L . \rho_{1}(x)=\rho_{2}(x)$ and $\forall a \in L$ and addresses $n \in\{\operatorname{base}(a), \ldots$, base $(a)+\operatorname{length}(a)-1\}$, $\mu_{1}(n)=\mu_{2}(n)$, respectively.

Definition 1 (Speculative Constant Time). A program $c$ is speculative constant time with respect to a security policy $L$, written $S C T_{L}(c)$, iff for all directives $D$ and initial configurations $C_{i}=$ $\left\langle[],[c], \mu_{i}, \rho_{i}\right\rangle$ for $i \in\{1,2\}$, if $C_{1} \approx_{L} C_{2}, C_{1} \Downarrow_{O_{1}}^{D} C_{1}^{\prime}$, and $C_{2} \Downarrow_{O_{2}}^{D} C_{2}^{\prime}$, then $O_{1}=O_{2}$ and $C_{1}^{\prime} \approx_{L} C_{2}^{\prime}$.

In the definition above, we consider syntactic equivalence of traces because both executions follow the same list of directives. We now present our soundness theorem: well-typed programs satisfy speculative constant-time. Our approach focuses on side-channel attacks through the observation trace and therefore relies on a separate, but standard, type system to control leaks through the program control-flow and architectural state. In particular, we write $C T_{L}(c)$ if $c$ follows the (sequential) constant time discipline from [Protzenko et al. 2019; Watt et al. 2019], i.e., it is free of secret-dependent branches and memory accesses.

Theorem 2 (Soundness). For all programs $c$ and security policies $L$, if $C T_{L}(c)$ and $\Gamma \vdash c$, then $\operatorname{SCT}_{L}(c)$.

As mentioned in Section 4, our transient flow-type system is oblivious to the security policy $L$, which is only required by the constant-time type system and the definition of speculative constant time.

We conclude with a corollary that combines all the components of our protection chain (type inference, type checking and automatic repair) and shows that repaired programs satisfy speculative constant time.

Corollary 1. For all sequential constant-time programs $C T_{L}(c)$, there exists a set of constraints $k$ such that $\Gamma^{*}$, Prot ${ }^{*} \vdash c \Rightarrow k$. Let $A$ be a set of variables that cut $k$. Then, it follows that $\operatorname{SCT}_{L}(\operatorname{repair}(c, A))$. 


\section{IMPLEMENTATION}

We implement BLADE as a compilation pass in the Cranelift [Bytecode Alliance 2020] Wasm codegenerator, which is used by the Lucet compiler and runtime [McMullen 2020]. BLADE first identifies all sources and sinks. Then, it finds the cut points using the Max-Flow/Min-Cut algorithm (§4.2), and either inserts fences at the cut points, or applies SLH to all of the loads which feed the cut point in the graph. This difference is why SLH sometimes requires code insertions in more locations.

Our SLH prototype implementation does not track the length of arrays, and instead uses a static constant for all array lengths when applying masking. Once compilers like Clang add support for conveying array length information to Wasm (e.g., via Wasm's custom section), our compilation pass would be able to take this information into account. This simplification in our experiments does not affect the sequence of instructions emitted for the SLH masks and thus BLADE's performance overhead is accurately measured.

Our Cranelift BLADE pass runs after the control-flow graph has been finalized and right before register allocation. ${ }^{13}$ Placing BLADE before register allocation allows our implementation to remain oblivious of low-level details such as register pressure and stack spills and fills. Ignoring the memory operations incurred by spills and fills simplifies BLADE's analysis and reduces the required number of protect statements. This, importantly, does not compromise the security of its mitigations: In Cranelift, spills and fills are always to constant addresses which are inaccessible to ordinary Wasm loads and stores, even speculatively. (Cranelift uses guard pages-not conditional bounds checks-to ensure that Wasm memory accesses cannot access anything outside the linear memory, such as the stack used for spills and fills.) As a result, we can treat stack spill slots like registers. Indeed, since BLADE runs before register allocation, it already traces def-use chains across operations that will become spills and fills. Even if a particular spill-fill sequence would handle potentially sensitive transient data, BLADE would insert a protect between the original transient source and the final transient sink (and thus mitigate the attack).

Our implementation implements a single optimization: we do not mark constant-address loads as transient sources. We assume that the program contains no loads from out-of-bounds constant addresses, and therefore that loads from constant (Wasm linear memory) addresses can never speculatively produce invalid data. As we describe below, however, we omit this optimization when considering Spectre v1.1.

At its core, our repair algorithm addresses Spectre v1 attacks based on PHT mispredictions. To also protect against Spectre variant 1.1 attacks, which exploit store forwarding in the presence of PHT mispredictions, ${ }^{14}$ we perform two additional mitigations. First, we mark constant-address loads as transient sources (and thus omit the above optimization). Under Spectre v1.1, a load from a constant address may speculatively produce transient data, if a previous speculative store wrote transient data to that constant address-and, thus, BLADE must account for this. Second, our SLH implementation marks all stored values as sinks, essentially preventing any transient data from being stored to memory. This is necessary when considering Spectre v1.1 because otherwise, ensuring that a load is in-bounds using SLH is insufficient to guarantee that the produced data is not transient-again, a previous speculative store may have written transient data to that in-bounds address.

\footnotetext{
${ }^{13}$ More precisely: The Cranelift register allocation pass modifies the control-flow graph as an initial step; we insert our pass after this initial step but before register allocation proper.

${ }^{14}$ Spectre v1 and Spectre v1.1 attacks are both classified as Spectre-PHT attacks [Canella et al. 2019].
} 


\section{EVALUATION}

We evaluate BlADE by answering two questions: (Q1) How many protects does BlADE insert when repairing existing programs? (Q2) What is the runtime performance overhead of eliminating speculative leaks with BLADE on existing hardware?

Benchmarks. We evaluate BLADE on existing cryptographic code taken from two sources. First, we consider two cryptographic primitives from CT-Wasm [Watt et al. 2019]:

$\triangleright$ The Salsa20 stream cipher, with a workload of 64 bytes.

$\triangleright$ The SHA-256 hash function, with workloads of 64 bytes (one block) or 8192 bytes (128 blocks).

Second, we consider automatically generated cryptographic primitives and protocols from the $\mathrm{HACL}^{*}$ [Zinzindohoué et al. 2017] library. We compile the automatically generated C code to Wasm using Clang's Wasm backend. (We do not use HACL*'s Wasm backend since it relies on a JavaScript embedding environment and is not well suited for Lucet.) Specifically, from HACL ${ }^{*}$ we consider:

$\Delta$ The ChaCha20 stream cipher, with a workload of 8192 bytes.

$\triangleright$ The Poly1305 message authentication code, with workloads of 1024 or 8192 bytes.

$\triangleright$ ECDH key agreement using Curve25519.

We selected these primitives to cover different kinds of modern crypto workloads (including hash functions, MACs, encryption ciphers, and public key exchange algorithms). We omitted primitives that had inline assembly or SIMD since Lucet does not yet support either; we also omitted the AES from HACL* and TEA from CT-Wasm-modern processors implement AES in hardware (largely because efficient software implementations of AES are generally not constant-time [Osvik et al. 2006]), while TEA is not used in practice. All the primitives we consider have been verified to be constant-time-free of cache and timing side-channels. However, the proofs assume a sequential execution model and do not account for speculative leaks as addressed in this work.

Experimental Setup. We conduct our experiments on an Intel Xeon Platinum 8160 (Skylake) with 1TB of RAM. The machine runs Arch Linux with kernel 5.8.14, and we use the Lucet runtime version $0.7 .0-\operatorname{dev}$ (Cranelift version 0.62 .0 with our modifications) compiled with rustc version 1.46. 0 . We collect benchmarks using the Rust criterion crate version 0.3 .3 [Heisler and Aparicio 2020] and report the point estimate for the mean runtime of each benchmark.

Reference and Baseline Comparisons. We compare BLADE to a reference (unsafe) implementation and a baseline (safe) implementation which simply protects every Wasm memory load instruction. We consider two baseline variants: The baseline solution with Spectre v1.1 mitigation protects every Wasm load instruction, while the baseline solution with only Spectre v1 mitigation protects only Wasm load instructions with non-constant addresses. The latter is similar to Clang's Spectre mitigation pass, which applies SLH to each non-constant array read [Carruth 2019]. We evaluate both BLADE and the baseline implementation with Spectre v1 protection and with both v1 and v1.1 protections combined. We consider both fence-based and SLH-based implementations of the protect primitive. In the rest of this section, we use Baseline-F and BLADE-F to refer to fence-based implementations of their respective mitigations and Baseline-S and BLADE-S to refer to the SLH-based implementations.

Results. Table 1 summarizes our results. With Spectre v1 protections, both BLADE-F and BLADE-S insert very few protects and have negligible performance overhead on most of our benchmarks-the geometric mean overheads imposed by BLADE-F and BLADE-S are $5.0 \%$ and $1.7 \%$, respectively. In contrast, the baseline passes insert between 3 and 1862 protections and incur significantly higher overheads than BLADE-the geometric mean overheads imposed by Baseline-F and Baseline-S are $80.2 \%$ and $19.4 \%$, respectively. 
Table 1. Ref: Reference implementation with no Spectre mitigations; Baseline-F: Baseline mitigation inserting fences; BLADE-F: BLADE using fences as protect; Baseline-S: Baseline mitigation using SLH; BLADE-S: BLADE using SLH; Overhead: Runtime overhead compared to Ref; Defs: number of fences inserted (Baseline-F and BLADE-F), or number of loads protected with SLH (Baseline-S and BLADE-S)

\begin{tabular}{|c|c|c|c|c|c|c|c|}
\hline \multirow[b]{2}{*}{ Benchmark } & \multirow[b]{2}{*}{ Defense } & \multicolumn{3}{|c|}{ Without v1.1 protections } & \multicolumn{3}{|c|}{ With v1.1 protections } \\
\hline & & Time & Overhead & Defs & Time & Overhead & Defs \\
\hline \multirow{5}{*}{ Salsa20 (CT-Wasm), 64 bytes } & Ref & 4.3 us & - & - & $4.3 \mathrm{us}$ & - & - \\
\hline & Baseline-F & 4.6 us & $7.2 \%$ & 3 & 8.6 us & $101.7 \%$ & 99 \\
\hline & BLADE-F & $4.4 \mathrm{us}$ & $1.9 \%$ & 0 & 4.3 us & $1.7 \%$ & 0 \\
\hline & Baseline-S & $4.4 \mathrm{us}$ & $2.7 \%$ & 3 & 5.3 us & $24.3 \%$ & 99 \\
\hline & BLADE-S & 4.3 us & $0.5 \%$ & 0 & 5.4 us & $26.4 \%$ & 99 \\
\hline \multirow{5}{*}{ SHA-256 (CT-Wasm), 64 bytes } & Ref & 13.7 us & - & - & 13.7 us & - & - \\
\hline & Baseline-F & 19.8 us & $43.8 \%$ & 23 & 20.3 us & $48.0 \%$ & 54 \\
\hline & BLADE-F & 13.8 us & $0.2 \%$ & 0 & 14.5 us & $5.4 \%$ & 3 \\
\hline & Baseline-S & 15.0 us & $9.1 \%$ & 23 & 15.1 us & $10.0 \%$ & 54 \\
\hline & BLADE-S & 13.9 us & $0.8 \%$ & 0 & 15.2 us & $10.9 \%$ & 54 \\
\hline \multirow{5}{*}{ SHA-256 (CT-Wasm), 8192 bytes } & Ref & 114.6 us & - & - & 114.6 us & - & - \\
\hline & Baseline-F & 516.6 us & $350.6 \%$ & 23 & 632.6 us & $451.8 \%$ & 54 \\
\hline & BLADE-F & 113.7 us & $-0.8 \%$ & 0 & 193.3 us & $68.6 \%$ & 3 \\
\hline & Baseline-S & 187.4 us & $63.4 \%$ & 23 & 208.0 us & $81.5 \%$ & 54 \\
\hline & BLADE-S & 115.2 us & $0.5 \%$ & 0 & 216.5 us & $88.9 \%$ & 54 \\
\hline \multirow{5}{*}{ ChaCha20 $\left(\mathrm{HACL}^{*}\right), 8192$ bytes } & Ref & $43.7 \mathrm{us}$ & - & - & 43.7 us & - & - \\
\hline & Baseline-F & $85.2 \mathrm{us}$ & $94.8 \%$ & 136 & 85.4 us & $95.3 \%$ & 142 \\
\hline & BLADE-F & $44.4 \mathrm{us}$ & $1.5 \%$ & 3 & 45.4 us & $3.8 \%$ & 7 \\
\hline & Baseline-S & 52.8 us & $20.8 \%$ & 136 & 53.3 us & $21.9 \%$ & 142 \\
\hline & BLADE-S & 43.6 us & $-0.3 \%$ & 3 & 53.8 us & $22.9 \%$ & 142 \\
\hline \multirow{5}{*}{ Poly1305 (HACL*), 1024 bytes } & Ref & 5.5 us & - & - & 5.5 us & - & - \\
\hline & Baseline-F & 6.3 us & $15.9 \%$ & 133 & 6.4 us & $17.2 \%$ & 139 \\
\hline & BLADE-F & 5.5 us & $1.4 \%$ & 3 & 5.6 us & $2.2 \%$ & 9 \\
\hline & Baseline-S & 5.6 us & $1.8 \%$ & 133 & 5.7 us & $4.4 \%$ & 139 \\
\hline & BLADE-S & 5.5 us & $1.0 \%$ & 3 & 5.6 us & $2.5 \%$ & 139 \\
\hline \multirow{5}{*}{ Poly1305 (HACL*), 8192 bytes } & Ref & $15.1 \mathrm{us}$ & - & - & $15.1 \mathrm{us}$ & - & - \\
\hline & Baseline-F & $21.3 \mathrm{us}$ & $41.1 \%$ & 133 & $21.4 \mathrm{us}$ & $41.2 \%$ & 139 \\
\hline & BLADE-F & $15.1 \mathrm{us}$ & $-0.0 \%$ & 3 & $15.2 \mathrm{us}$ & $0.8 \%$ & 9 \\
\hline & Baseline-S & $16.2 \mathrm{us}$ & $7.2 \%$ & 133 & 16.3 us & $7.6 \%$ & 139 \\
\hline & BLADE-S & 15.2 us & $0.7 \%$ & 3 & $16.2 \mathrm{us}$ & $7.1 \%$ & 139 \\
\hline \multirow{5}{*}{ ECDH Curve25519 (HACL*) } & Ref & 354.3 us & - & - & 354.3 us & - & - \\
\hline & Baseline-F & 989.8 us & $179.3 \%$ & 1862 & 1006.4 us & $184.0 \%$ & 1887 \\
\hline & BLADE-F & 479.9 us & $35.4 \%$ & 235 & 497.8 us & $40.5 \%$ & 256 \\
\hline & Baseline-S & 507.0 us & $43.1 \%$ & 1862 & 520.4 us & $46.9 \%$ & 1887 \\
\hline & BLADE-S & 386.1 us & $9.0 \%$ & 1419 & 516.8 us & $45.9 \%$ & 1887 \\
\hline \multirow{5}{*}{ Geometric means } & Ref & & - & & & - & \\
\hline & Baseline-F & & $80.2 \%$ & & & $104.8 \%$ & \\
\hline & BLADE-F & & $5.0 \%$ & & & $15.3 \%$ & \\
\hline & Baseline-S & & $19.4 \%$ & & & $25.8 \%$ & \\
\hline & BLADE-S & & $1.7 \%$ & & & $26.6 \%$ & \\
\hline
\end{tabular}

With both v1 and v1.1 protections, BLADE-F inserts an order of magnitude fewer protections than Baseline-F, and has correspondingly low performance overhead-the geometric mean overhead of BLADE-F is $15.3 \%$, whereas Baseline-F's is $104.8 \%$. The geometric mean overhead of both BLADE-S 
and Baseline-S, on the other hand, is roughly $26 \%$. Unlike BLADE-F, BLADE-S must mark all stored values as sinks in order to eliminate Spectre v1.1 attacks; for these benchmarks, this countermeasure requires BLADE-S to apply protections to every Wasm load, just like Baseline-S. Indeed, we see in the table that Baseline-S and BLADE-S make the exact same number of additions to the code.

We make three observations from our measurements. First, and somewhat surprisingly, BLADE does not insert any protects for Spectre v1 on any of the CT-Wasm benchmarks. We attribute this to the style of code: the CT-Wasm primitives are hand-written and, moreover, statically allocate variables and arrays in the Wasm linear memory-which, in turn, results in many constant-address loads. This is unlike the $\mathrm{HACL}^{*}$ primitives which are written in $\mathrm{F}^{*}$, compiled to $\mathrm{C}$ and then Wasmand thus require between 3 and 235 protects.

Second, the benchmarks with short reference runtimes tend to have overall lower overheads, particularly for the baseline schemes. This is because for short workloads, the overall runtime is dominated by sandbox setup and teardown, which BLADE does not introduce much overhead for. In contrast, for longer workloads, the execution of the Wasm code becomes the dominant portion of the benchmark-and exposes the overhead imposed by the different mitigations. We explore the relationship between workload size and performance overhead in more detail in [Vassena et al. 2020].

And third, we observe for the Spectre v1 version that SLH gives overall better performance than fences, as expected. This is true even in the case of Curve25519, where implementing protect using SLH (BLADE-S) results in a significant increase in the number of protections versus the fence-based implementation (BLADE-F). Even in this case, the more targeted restriction of speculation, and the less heavyweight impact on the pipeline, allows SLH to still prevail over the fewer fences. However, this advantage is lost when considering both v1 and v1.1 mitigation: The sharp increase in the number of protects required for v1.1 ends up being slower than using (fewer) fences. A hybrid approach that uses both fences and SLH could potentially outperform both BLADE-F and BLADE-S.

In reality, though, both versions are inadequate software emulations of what the protect primitive should be. Fences take a heavy toll on the pipeline and are far too restrictive of speculation, while SLH pays a heavy instruction overhead for each instance, and can only be applied directly to loads, not to arbitrary cut points. A hardware implementation of the protect primitive could combine the best of BLADE-F and BLADE-S: targeted restriction of speculation, minimal instruction overhead, and only as many defenses as BLADE-F, without the inflation in insertion count required by BLADE-S.

However, even without any hardware assistance, both versions of the BLADE tool provide significant performance gains over the current state of the art in mitigating Spectre v1, and over existing fence-based solutions when targeting v1 or both v1 and v1.1.

\section{RELATED WORK}

Speculative Execution Semantics. Several semantics models for speculative execution have been proposed recently [Cauligi et al. 2020; Cheang et al. 2019; Disselkoen et al. 2019; Guanciale et al. 2020; Guarnieri et al. 2020; McIlroy et al. 2019]. Of those, [Cauligi et al. 2020] is closest to ours, and inspired our semantics (e.g., we share the 3-stages pipeline, attacker-supplied directives and the instruction reorder buffer). However, their semantics-and, indeed, the semantics of most of the other works-are exclusively for low-level assembly-like languages ${ }^{15}$ In contrast, our JIT semantics bridges the gap between high-level commands and low-level instructions, which allows us to reason about speculative execution of source-level imperative programs through straightforward typing rules, while being faithful to low-level microarchitectural details. Moreover, the idea of modeling

\footnotetext{
${ }^{15}$ The one exception, Disselkoen et al. [2019], present a Spectre-aware relaxed memory model based on pomsets, which is even further abstracted from the microarchitectural features of real processors.
} 
speculative and out-of-order execution using stacks of progressively flattened commands is novel and key to enable source-level reasoning about the low-level effects of speculation.

Detection and Repair. Wu and Wang [2019] detect cache side channels via abstract interpretation by augmenting the program control-flow to accommodate for speculation. SPECTECTOR [Guarnieri et al. 2020] and Pitchfork [Cauligi et al. 2020] use symbolic execution on x86 binaries to detect speculative vulnerabilities. Cheang et al. [2019] and Bloem et al. [2019] apply bounded model checking to detect potential speculative vulnerabilities respectively via 4-ways self-composition and taint-tracking. These efforts assume a fixed speculation bound, and they focus on vulnerability detection rather than proposing techniques to repair vulnerable programs. Furthermore, many of these works consider only in-order execution. In contrast, our type system enforces speculative constant-time when program instructions are executed out-of-order with unbounded speculationand our tool BLADE automatically synthesizes repairs. Separately, oo7 [Wang et al. 2018] statically analyzes a binary from a set of untrusted input sources, detecting vulnerable patterns and inserting fences in turn. Our tool, BLADE, not only repairs vulnerable programs without user annotation, but ensures that program patches contain a minimum number of fences. Furthermore, BLADE formally guarantees that repaired programs are free from speculation-based attacks.

Concurrent to our work, Intel proposed a mitigation for a new class of LVI attacks [Intel 2020; Van Bulck et al. 2020]. Like BLADE, they implement a compiler pass that analyzes the program to determine an optimal placement of fences to cut source-to-sink data flows. While we consider an abstract, ideal protect primitive, they focus on the optimal placement of fences in particular. This means that they optimize the fence placement by taking into account the coarse-grained effects of fences-e.g., one fence providing a speculation barrier for multiple independent data-dependency chains. ${ }^{16}$ This also means, however, their approach does not easily transfer to using SLH for cases where SLH would be faster.

Hardware-based Mitigations. To eliminate speculative attacks, several secure hardware designs have been proposed. Taram et al. [2019] propose context-sensitive fencing, a hardware-based mitigation that dynamically inserts fences in the instruction stream when dangerous conditions arise. InvisiSpec [Yan et al. 2018] features a special speculative buffer to prevent speculative loads from polluting the cache. STT [Yu et al. 2019] tracks speculative taints dynamically inside the processor micro-architecture and stalls instructions to prevent speculative leaks. Schwarz et al. [2020] propose ConTExT, a whole architecture change (applications, compilers, operating systems, and hardware) to eliminate all Spectre attacks. Though BLADE can benefit from a hardware implementation of protect, this work also shows that Spectre-PHT on existing hardware can be automatically eliminated in pure software with modest performance overheads.

\section{LIMITATIONS AND FUTURE WORK}

BLADE only addresses Spectre-PHT attacks and does so at the Wasm-layer. Extending BLADE to tackle other Spectre variants and the limitations of operating on Wasm is future work.

Other Spectre Variants. The Spectre-BTB variant [Kocher et al. 2019] mistrains the Branch Target Buffer (BTB), which is used to predict indirect jump targets, to hijack the (speculative) control-flow of the program. Although Wasm does not provide an unrestricted indirect jump instruction, the indirect function call instruction-which is used to call functions registered in a function table-can be abused by an attacker. To address (in-process) Spectre-BTB, we could extend our type system to restrict the values used as indices into the function table to be typed as stable.

\footnotetext{
${ }^{16}$ Unlike our approach, their resulting optimization problem is NP-hard-and only sub-optimal solutions may be found through heuristics.
} 
The other Spectre variant, Spectre-RSB [Koruyeh et al. 2018; Maisuradze and Rossow 2018], abuses the return stack buffer. To mitigate these attacks, we could analyze Wasm code to identify potential RSB over/underflows and insert fences in response, or use mitigation strategies like RSB stuffing [Intel 2018b]. A more promising approach, however, is to use Intel's recent shadow stack, which ensures that returns cannot be speculatively hijacked [Shanbhogue et al. 2019].

Detecting Spectre Gadgets at the Binary Level. BLADE operates on Wasm code-or more precisely, on the Cranelift compiler's IR-and can thus miss leaks inserted by the compiler passes that run after BLADE-namely, register allocation and instruction selection. Though these passes are unlikely to introduce such leaks, we leave the validation of the generated binary code to future work.

Spectre Resistant Compilation. An alternative to repairing existing programs is to ensure they are compiled securely from the start. Recent works have developed verified constant-time-preserving optimizing compilers for generating correct, efficient, and secure cryptographic code [Almeida et al. 2017; Barthe et al. 2019]. Doing this for speculative constant-time, and understanding which optimizations break the SCT notion, is an interesting direction for future work.

Bounds Information. BLADE-S relies on array bounds information to implement the speculative load hardening. For a memory safe language, this information can be made available to BLADE when compiling to Wasm (e.g., as a custom section). When compiling languages like $\mathrm{C}$, where arrays bounds information is not explicit, this is harder-and we would need to use program analysis to track array lengths statically [Venet and Brat 2004]. Although such an analysis may be feasible for cryptographic code, it is likely to fall short for other application domains (e.g., due to dynamic memory allocation and pointer chasing). In these cases, we could track array lengths at runtime (e.g., by instrumenting programs [Nagarakatte et al. 2009]) or, more simply, fall back to fences (especially since the overhead of tracking bounds information at runtime is typically high).

\section{CONCLUSION}

We presented BLADE, a fully automatic approach to provably and efficiently eliminate speculationbased leakage in unannotated cryptographic code. BLADE statically detects data flows from transient sources to stable sinks and synthesizes a minimal number of fence-based or SLH-based protect calls to eliminate potential leaks. Our evaluation shows that BLADE inserts an order of magnitude fewer protections than would be added by today's compilers, and that existing crypto primitives repaired with BLADE impose modest overheads when using both fences and SLH for protect.

\section{ACKNOWLEDGEMENTS}

We thank the reviewers and our shepherd Aseem Rastogi for their suggestions and insightful comments. Many thanks to Shravan Narayan, Ravi Sahita, and Anjo Vahldiek-Oberwagner for fruitful discussions. This work was supported in part by gifts from Fastly, Fujitsu, and Cisco; by the NSF under Grant Number CNS-1514435 and CCF-1918573; by ONR Grant N000141512750; by the German Federal Ministry of Education and Research (BMBF) through funding for the CISPAStanford Center for Cybersecurity; and, by the CONIX Research Center, one of six centers in JUMP, a Semiconductor Research Corporation (SRC) program sponsored by DARPA.

\section{REFERENCES}

Alex Aiken. 1996. Constraint-based program analysis. In Static Analysis, Radhia Cousot and David A. Schmidt (Eds.). Springer Berlin Heidelberg, Berlin, Heidelberg, 1-1.

José Bacelar Almeida, Manuel Barbosa, Gilles Barthe, Arthur Blot, Benjamin Grégoire, Vincent Laporte, Tiago Oliveira, Hugo Pacheco, Benedikt Schmidt, and Pierre-Yves Strub. 2017. Jasmin: High-Assurance and High-Speed Cryptography. In Proceedings of the 2017 ACM SIGSAC Conference on Computer and Communications Security (Dallas, Texas, USA) (CCS '17). Association for Computing Machinery, New York, NY, USA, 1807-1823. https://doi.org/10.1145/3133956.3134078 
Jose Bacelar Almeida, Manuel Barbosa, Gilles Barthe, François Dupressoir, and Michael Emmi. 2016. Verifying Constant-Time Implementations. In 25th USENIX Security Symposium (USENIX Security 16). USENIX Association, Austin, TX, 53-70. https://www.usenix.org/conference/usenixsecurity16/technical-sessions/presentation/almeida

AMD. 2018. Software Techniques For Managing Speculation On AMD Processors. https://developer.amd.com/wpcontent/resources/Managing-Speculation-on-AMD-Processors.pdf.

Gilles Barthe, Sandrine Blazy, Benjamin Grégoire, Rémi Hutin, Vincent Laporte, David Pichardie, and Alix Trieu. 2019. Formal Verification of a Constant-Time Preserving C Compiler. Proc. ACM Program. Lang. 4, POPL, Article 7 (Dec. 2019), 30 pages. https://doi.org/10.1145/3371075

Atri Bhattacharyya, Alexandra Sandulescu, Matthias Neugschwandtner, Alessandro Sorniotti, Babak Falsafi, Mathias Payer, and Anil Kurmus. 2019. SMoTherSpectre: Exploiting Speculative Execution Through Port Contention. In Proceedings of the 2019 ACM SIGSAC Conference on Computer and Communications Security (London, United Kingdom) (CCS '19). ACM, New York, NY, USA, 785-800. https://doi.org/10.1145/3319535.3363194

Roderick Bloem, Swen Jacobs, and Yakir Vizel. 2019. Efficient Information-Flow Verification Under Speculative Execution. In Automated Technology for Verification and Analysis, Yu-Fang Chen, Chih-Hong Cheng, and Javier Esparza (Eds.). Springer International Publishing, Cham, 499-514.

Google Security Blog. 2010. Mitigating Spectre with Site Isolation in Chrome. https://security.googleblog.com/2018/07/ mitigating-spectre-with-site-isolation.html.

Bytecode Alliance. 2020. Cranelift Code Generator. https://github.com/bytecodealliance/wasmtime/tree/main/cranelift.

Claudio Canella, Jo Van Bulck, Michael Schwarz, Moritz Lipp, Benjamin Von Berg, Philipp Ortner, Frank Piessens, Dmitry Evtyushkin, and Daniel Gruss. 2019. A Systematic Evaluation of Transient Execution Attacks and Defenses. In Proceedings of the 28th USENIX Conference on Security Symposium (Santa Clara, CA, USA) (SEC'19). USENIX Association, Berkeley, CA, USA, 249-266. http://dl.acm.org/citation.cfm?id=3361338.3361356

Chandler Carruth. 2019. Speculative Load Hardening. https://llvm.org/docs/SpeculativeLoadHardening.html.

Sunjay Cauligi, Craig Disselkoen, Klaus von Gleissenthall, Dean Tullsen, Deian Stefan, Tamara Rezk, and Gilles Barthe. 2020. Constant-Time Foundations for the New Spectre Era. In Proc. ACM Conference on Programming Language Design and Implementation.

Sunjay Cauligi, Gary Soeller, Brian Johannesmeyer, Fraser Brown, Riad S. Wahby, John Renner, Benjamin Gregoire, Gilles Barthe, Ranjit Jhala, and Deian Stefan. 2019. FaCT: A DSL for timing-sensitive computation. In Programming Language Design and Implementation (PLDI). ACM SIGPLAN.

Kevin Cheang, Cameron Rasmussen, Sanjit A. Seshia, and Pramod Subramanyan. 2019. A Formal Approach to Secure Speculation. In Proceedings of the Computer Security Foundations Symposium (CSF).

Craig Disselkoen, Radha Jagadeesan, Alan Jeffrey, and James Riely. 2019. The Code That Never Ran: Modeling Attacks on Speculative Evaluation. In 2019 IEEE Symposium on Security and Privacy, SP 2019, San Francisco, CA, USA, May 19-23, 2019. 1238-1255. https://doi.org/10.1109/SP.2019.00047

Daniel Donenfeld. 2020. More Spectre mitigations in MSVC. https://devblogs.microsoft.com/cppblog/more-spectremitigations-in-msvc/.

D. R. Ford and D. R. Fulkerson. 2010. Flows in Networks. Princeton University Press, USA.

Qian Ge, Yuval Yarom, David Cock, and Gernot Heiser. 2018. A survey of microarchitectural timing attacks and countermeasures on contemporary hardware. In fournal of Cryptographic Engineering.

Roberto Guanciale, Musard Balliu, and Mads Dam. 2020. InSpectre: Breaking and Fixing Microarchitectural Vulnerabilities by Formal Analysis. In Proceedings of the 2020 ACM SIGSAC Conference on Computer and Communications Security (Virtual Event, USA) (CCS '20). Association for Computing Machinery, New York, NY, USA, 1853-1869. https://doi.org/ $10.1145 / 3372297.3417246$

Marco Guarnieri, Boris Koepf, José Francisco Morales, Jan Reineke, and Andrés Sánchez. 2020. Spectector: Principled Detection of Speculative Information Flows. In Proc. IEEE Symp. on Security and Privacy (SSP '20).

Andreas Haas, Andreas Rossberg, Derek L. Schuff, Ben L. Titzer, Michael Holman, Dan Gohman, Luke Wagner, Alon Zakai, and JF Bastien. 2017. Bringing the Web up to Speed with WebAssembly. In Proceedings of the 38th ACM SIGPLANConference on Programming Language Design and Implementation (Barcelona, Spain) (PLDI 2017). Association for Computing Machinery, New York, NY, USA, 185-200. https://doi.org/10.1145/3062341.3062363

Brook Heisler and Jorge Aparicio. 2020. Criterion.rs: Statistics-driven Microbenchmarking in Rust. https://crates.io/crates/ criterion.

Jann Horn. 2018. speculative execution, variant 4: speculative store bypass. https://bugs.chromium.org/p/project-zero/ issues/detail?id=1528.

Intel. 2018a. Intel Analysis of Speculative Execution Side Channels. https://newsroom.intel.com/wp-content/uploads/sites/ 11/2018/01/Intel-Analysis- of-Speculative-Execution-Side-Channels.pdf.

Intel. 2018b. Retpoline: A Branch Target Injection Mitigation. https://software.intel.com/security-software-guidance/apiapp/sites/default/files/Retpoline-A-Branch-Target-Injection-Mitigation.pdf. 
Intel. 2020. An Optimized Mitigation Approach for Load Value Injection. https://software.intel.com/security-softwareguidance/insights/optimized-mitigation-approach-load-value-injection.

Vladimir Kiriansky and Carl Waldspurger. 2018. Speculative Buffer Overflows: Attacks and Defenses. CoRR abs/1807.03757 (2018). arXiv:1807.03757 http://arxiv.org/abs/1807.03757

Paul Kocher, Jann Horn, Anders Fogh, , Daniel Genkin, Daniel Gruss, Werner Haas, Mike Hamburg, Moritz Lipp, Stefan Mangard, Thomas Prescher, Michael Schwarz, and Yuval Yarom. 2019. Spectre Attacks: Exploiting Speculative Execution. In 40th IEEE Symposium on Security and Privacy (S\&P'19).

Esmaeil Mohammadian Koruyeh, Khaled N. Khasawneh, Chengyu Song, and Nael Abu-Ghazaleh. 2018. Spectre Returns! Speculation Attacks Using the Return Stack Buffer. In Proceedings of the 12th USENIX Conference on Offensive Technologies (Baltimore, MD, USA) (WOOT'18). USENIX Association, Berkeley, CA, USA, 3-3. http://dl.acm.org/citation.cfm?id= 3307423.3307426

J. Landauer and T. Redmond. 1993. A lattice of information. In [1993] Proceedings Computer Security Foundations Workshop VI. 65-70. https://doi.org/10.1109/CSFW.1993.246638

Moritz Lipp, Michael Schwarz, Daniel Gruss, Thomas Prescher, Werner Haas, Anders Fogh, Jann Horn, Stefan Mangard, Paul Kocher, Daniel Genkin, Yuval Yarom, and Mike Hamburg. 2018. Meltdown: Reading Kernel Memory from User Space. In 27th USENIX Security Symposium (USENIX Security 18).

Giorgi Maisuradze and Christian Rossow. 2018. Ret2Spec: Speculative Execution Using Return Stack Buffers. In Proceedings of the 2018 ACM SIGSAC Conference on Computer and Communications Security (Toronto, Canada) (CCS '18). ACM, New York, NY, USA, 2109-2122. https://doi.org/10.1145/3243734.3243761

Ross McIlroy, Jaroslav Sevcík, Tobias Tebbi, Ben L. Titzer, and Toon Verwaest. 2019. Spectre is here to stay: An analysis of side-channels and speculative execution. CoRR abs/1902.05178 (2019). arXiv:1902.05178 http://arxiv.org/abs/1902.05178

Tyler McMullen. 2020. Lucet: A Compiler and Runtime for High-Concurrency Low-Latency Sandboxing. Principles of Secure Compilation (PriSC).

Daniel Moghimi, Moritz Lipp, Berk Sunar, and Michael Schwarz. 2020. Medusa: Microarchitectural Data Leakage via Automated Attack Synthesis. In 29th USENIX Security Symposium (USENIX Security 20). USENIX Association, Boston, MA. https://www.usenix.org/conference/usenixsecurity20/presentation/moghimi-medusa

Mozilla Wiki 2018. Security/Sandbox. https://wiki.mozilla.org/Security/Sandbox.

A. C. Myers, A. Sabelfeld, and S. Zdancewic. 2004. Enforcing robust declassification. In Proceedings. 17th IEEE Computer Security Foundations Workshop, 2004. 172-186. https://doi.org/10.1109/CSFW .2004.1310740

Santosh Nagarakatte, Jianzhou Zhao, Milo M.K. Martin, and Steve Zdancewic. 2009. SoftBound: Highly Compatible and Complete Spatial Memory Safety for c. In Proceedings of the 30th ACM SIGPLAN Conference on Programming Language Design and Implementation (Dublin, Ireland) (PLDI '09). Association for Computing Machinery, New York, NY, USA, 245-258. https://doi.org/10.1145/1542476.1542504

Hanne Riis Nielson and Flemming Nielson. 1998. Flow logics for constraint based analysis. In Compiler Construction, Kai Koskimies (Ed.). Springer Berlin Heidelberg, Berlin, Heidelberg, 109-127.

Dag Arne Osvik, Adi Shamir, and Eran Tromer. 2006. Cache attacks and countermeasures: the case of AES. In Proceedings of the 2006 The Cryptographers' Track at the RSA conference on Topics in Cryptology (CT-RSA'06). Springer-Verlag.

Andrew Pardoe. 2018. Spectre mitigations in MSVC. https://devblogs.microsoft.com/cppblog/spectre-mitigations-in-msvc/.

Jonathan Protzenko, Benjamin Beurdouche, Denis Merigoux, and Karthikeyan Bhargavan. 2019. Formally Verified Cryptographic Web Applications in WebAssembly. In Security and Privacy.

Charles Reis, Alexander Moshchuk, and Nasko Oskov. 2019. Site Isolation: Process Separation for Web Sites within the Browser. In USENIX Security Symposium.

Michael Schwarz, Moritz Lipp, Claudio Canella, Robert Schilling, Florian Kargl, and Daniel Gruss. 2020. ConTExT: A Generic Approach for Mitigating Spectre. In Proc. Network and Distributed System Security Symposium. https: //doi.org/10.14722/ndss.2020.24271

Vedvyas Shanbhogue, Deepak Gupta, and Ravi Sahita. 2019. Security Analysis of Processor Instruction Set Architecture for Enforcing Control-Flow Integrity. In International Workshop on Hardware and Architectural Support for Security and Privacy (HASP).

Mohammadkazem Taram, Ashish Venkat, and Dean Tullsen. 2019. Context-Sensitive Fencing: Securing Speculative Execution via Microcode Customization. In Proceedings of the Twenty-Fourth International Conference on Architectural Support for Programming Languages and Operating Systems (Providence, RI, USA) (ASPLOS '19). Association for Computing Machinery, New York, NY, USA, 395-410. https://doi.org/10.1145/3297858.3304060

Vadim Tkachenko. 2018. 20-30\% Performance Hit from the Spectre Bug Fix on Ubuntu. https://www.percona.com/blog/2018/01/23/20-30-performance-hit-spectre-bug-fix-ubuntu/.

Eran Tromer, Dag Arne Osvik, and Adi Shamir. 2010. Efficient Cache Attacks on AES, and Countermeasures. f. Cryptol. 23 , 1 (Jan. 2010), 37-71. https://doi.org/10.1007/s00145-009-9049-y 
Jo Van Bulck, Daniel Moghimi, Michael Schwarz, Moritz Lipp, Marina Minkin, Daniel Genkin, Yarom Yuval, Berk Sunar, Daniel Gruss, and Frank Piessens. 2020. LVI: Hijacking Transient Execution through Microarchitectural Load Value Injection. In 41th IEEE Symposium on Security and Privacy (S\&P'20).

Marco Vassena, Craig Disselkoen, Klaus V. Gleissenthall, Sunjay Cauligi, Rami Gökhan Kici, Ranjit Jhala, Dean Tullsen, and Deian Stefan. 2020. Automatically Eliminating Speculative Leaks from Cryptographic Code with Blade. CoRR abs/2005.00294 (2020). arXiv:2005.00294 https://arxiv.org/abs/2005.00294

Arnaud Venet and Guillaume Brat. 2004. Precise and Efficient Static Array Bound Checking for Large Embedded C Programs. SIGPLAN Not. 39, 6 (June 2004), 231-242. https://doi.org/10.1145/996893.996869

D. Volpano, G. Smith, and C. Irvine. 1996. A Sound Type System for Secure Flow Analysis. F. Computer Security 4, 3 (1996), 167-187.

Guanhua Wang, Sudipta Chattopadhyay, Ivan Gotovchits, Tulika Mitra, and Abhik Roychoudhury. 2018. oo7: Lowoverhead Defense against Spectre Attacks via Binary Analysis. CoRR abs/1807.05843 (2018). arXiv:1807.05843 http: //arxiv.org/abs/1807.05843

Conrad Watt, John Renner, Natalie Popescu, Sunjay Cauligi, and Deian Stefan. 2019. CT-wasm: Type-driven Secure Cryptography for the Web Ecosystem. Proc. ACM Program. Lang. 3, POPL, Article 77 (Jan. 2019), 29 pages. https: //doi.org/10.1145/3290390

Meng Wu and Chao Wang. 2019. Abstract Interpretation Under Speculative Execution. In Proceedings of the 40th ACM SIGPLAN Conference on Programming Language Design and Implementation (Phoenix, AZ, USA) (PLDI 2019). ACM, New York, NY, USA, 802-815. https://doi.org/10.1145/3314221.3314647

Mengjia Yan, Jiho Choi, Dimitrios Skarlatos, Adam Morrison, Christopher W. Fletcher, and Josep Torrellas. 2018. InvisiSpec: Making Speculative Execution Invisible in the Cache Hierarchy. In Proceedings of the 51st Annual IEEE/ACM International Symposium on Microarchitecture (Fukuoka, Japan) (MICRO-51). IEEE Press, Piscataway, NJ, USA, 428-441. https: //doi.org/10.1109/MICRO.2018.00042

Yuval Yarom and Katrina Falkner. 2014. FLUSH+RELOAD: A High Resolution, Low Noise, L3 Cache Side-Channel Attack. In 23rd USENIX Security Symposium (USENIX Security 14). USENIX Association, San Diego, CA, 719-732. https: //www.usenix.org/conference/usenixsecurity14/technical-sessions/presentation/yarom

Jiyong Yu, Mengjia Yan, Artem Khyzha, Adam Morrison, Josep Torrellas, and Christopher W. Fletcher. 2019. Speculative Taint Tracking (STT): A Comprehensive Protection for Speculatively Accessed Data. In Proceedings of the 52nd Annual IEEE/ACM International Symposium on Microarchitecture, MICRO 2019, Columbus, OH, USA, October 12-16, 2019. 954-968. https://doi.org/10.1145/3352460.3358274

Jean-Karim Zinzindohoué, Karthikeyan Bhargavan, Jonathan Protzenko, and Benjamin Beurdouche. 2017. HACL * : A Verified Modern Cryptographic Library. In ACM Conference on Computer and Communications Security (CCS). Dallas, United States. https://hal.inria.fr/hal-01588421 